\title{
Das Gefängnis als Entwicklungsraum
}

\section{Ein Plädoyer für eine erweiterte Perspektive auf den Justizvollzug}

\author{
Klaus-Peter Dahle ${ }^{1}$. Werner Greve ${ }^{1}$. Daniela Hosser ${ }^{2}$. Thomas Bliesener ${ }^{3}$ \\ Eingegangen: 8. Dezember 2019 / Angenommen: 17. Dezember 2019 / Online publiziert: 22. Januar 2020 \\ (c) Der/die Autor(en) 2020
}

\section{Zusammenfassung}

Der Justizvollzug sieht sich zunehmenden Herausforderungen gegenüber. So verändert sich einerseits ihre Klientel in Richtung eines multikulturellen Schmelztiegels, was neue Phänomene mit sich bringt, wie z. B. Radikalisierungsprozesse oder kriminogene und organisierte Clanstrukturen. Es findet sich aber auch eine wachsende Kerngruppe im Vollzug gealterter Menschen mit vielfach gescheiterten Behandlungsversuchen, langer Haftsozialisation, oft psychopathologischer Vorbelastung und mit ungünstiger Entlassungsperspektive. Auf der anderen Seite erteilten die Bundesländer den Anstalten zunehmend den Auftrag, ihre Ressourcen verstärkt auf kriminogene Hochrisikogruppen zu fokussieren, und der Bundesgesetzgeber legte ihnen überdies besondere Behandlungspflichten für Sicherungsverwahrte und für die von dieser Maßregel bedrohte Gruppe von Gefangenen auf. Es mehren sich die Indizien, dass die Gefängnisse mit ihren traditionellen Mitteln bei der Bewältigung dieser Aufgaben an Grenzen stoßen. Anliegen des vorliegenden Beitrags ist es deshalb, dafür zu werben, die traditionellen Mittel einer primär erzieherisch und therapeutisch gedachten Intervention auszuweiten und in Richtung einer stärker grundsätzlichen Betrachtung der Voraussetzungen und Prozesse für Veränderungen in dieser speziellen Institution zu erweitern. Nach Überzeugung der Autoren bietet hierfür die Entwicklungspsychologie gute Voraussetzungen und ein passendes methodisches und theoretisches Rüstzeug.

Im Beitrag wird deshalb der Justizvollzug zunächst historisch analysiert und sein Auftrag zur Resozialisierung seiner Klientel als Auftrag zur Entwicklungsintervention interpretiert. Dies bietet die Argumentationsgrundlage dafür, eine entwicklungspsychologische Perspektive auf Veränderungsprozesse der Gefangenen einzunehmen, wobei es nicht um eine spezielle Entwicklungspsychologie des Strafvollzuges und ihrer Klientel geht, sondern vielmehr um eine entwicklungspsychologisch fundierte Betrachtung; mithin um eine Entwicklungspsychologie im Strafvollzug. Im zweiten Teil wird der Versuch unternommen, den Rahmen für eine mögliche entwicklungspsychologische Analyse des Justizvollzuges und seiner Klientel zu entwerfen. Dabei wird davon ausgegangen, dass sich Veränderung entlang der Beschäftigung des Individuums mit Problemen vollzieht, die sich ihm im Lauf seiner Biografie stellen. Deshalb werden zunächst typische Aufgaben betrachtet, mit denen ein Mensch im Lauf seines Lebens konfrontiert wird, mit Blick auf etwaige Besonderheiten einer straffälligen Personengruppe. Weiterhin werden außergewöhnliche, „kritische“ Lebensereignisse untersucht, die wegen ihrer Setzung veränderter Rahmenbedingungen Veränderungserfordernisse bedingen. Dabei wird argumentiert, dass die spezielle Klientel im Kontext einer Haftanstalt vermehrte Risiken solcher kritischen Lebensereignisse birgt. Schließlich werden Probleme aus einer aktionalen Entwicklungsperspektive, insbesondere selbst gesetzter Lebensziele, analysiert. Dabei werden die Prozesse, mit denen Menschen auf solche Herausforderungen reagieren, einer näheren Betrachtung unterzogen. Auch hier wird deutlich, dass die erforderlichen Regulationsprozesse bei einer strafgefangenen Population Besonderheiten erwarten lassen, die, zu untersuchen, lohnenswert zu sein verspricht. Schließlich wird der Strafvollzug als Raum, in dem sich Veränderungsprozesse vollziehen sollen, einer entwicklungspsychologisch informierten Analyse unterzogen. Es zeigt sich, dass eine entwicklungspsychologische Erweiterung der Perspektive auf den Justizvollzug in Praxis und Forschung Potenziale bereithält, zu einer Verbreiterung der Möglichkeiten erfolgversprechender Interventionen beizutragen.

$\triangle$ Prof. Dr. Klaus-Peter Dahle

klauspeter.dahle@uni-hildesheim.de

1 Stiftung Universität Hildesheim, Hildesheim, Deutschland
2 Technische Universität Braunschweig, Braunschweig, Deutschland

3 Kriminologisches Forschungsinstitut Niedersachsen, Hannover, Deutschland 
Schlüsselwörter Strafvollzug · Resozialisierung · Straftäterbehandlung · Entwicklungspsychologie · Entwicklungsregulation

\title{
The prison as a space for development
}

A plea for a broader developmental perspective on the correctional system

\begin{abstract}
The penal system is facing increasing challenges. On the one hand, the clientele is changing in the direction of a multicultural melting pot accompanied by new phenomena, such as radicalization processes and organized clan structures. Furthermore, there is an increasing group of inmates who have grown old in prison and are characterized by many failed attempts at treatment, long periods of imprisonment, often psychopathological disorders and unfavorable perspectives for release. On the other hand, the federal states in Germany have increasingly mandated the institutions to focus the resources on criminogenic high-risk groups. In addition, the federal legislature imposed on them special treatment tasks for preventive detention centers and for the group of prisoners threatened by this measure. There is growing evidence that prisons are reaching their limits in coping with these challenges with their traditional means. The aim of this article is therefore to promote the expansion of the traditional means of intervention primarily intended for educational and therapeutic purposes, with which the resocialization mandate to the penal system has been implemented so far. Another aim is to expand them in the direction of a more fundamental consideration of the prerequisites and processes for changes in correctional institutions. The authors are convinced that developmental psychology offers promising prerequisites and appropriate methodological and theoretical tools.

In the article the prison system is first analyzed historically and its mandate to resocialize the clientele is interpreted as a mandate for developmental intervention. This provides the basis for the argumentation to take a developmentally psychologically sound perspective on the processes of change of prisoners in prison. This does not entail a particular developmental psychology of the prison system itself but rather of a developmentally psychologically informed consideration and thus of a developmental psychology within the prison system. In the second part an attempt is made to design the framework for a possible developmental psychological analysis of the prison system and its clientele. It is assumed that change, i.e. development, takes place along the individual's responses to and coping with problems that a person faces in the course of the biography. For this reason, typical tasks with which a person is confronted in the course of life are first considered, albeit with a view to special features of a target group of criminal offenders. Furthermore, extraordinary "critical" life events are examined, which cause the requirement for change due to their altering of basic conditions for the individual's development. It is argued that the special clientele in the special context of a prison holds increased risks of such critical life events. Finally, problems are analyzed from an actional perspective on human development in general and the perspective of self-selected life goals in particular. When the processes which regulate peoples' responses to their developmental tasks and challenges are examined, it can be seen that the necessary regulation processes within a prison population can be expected to have special characteristics that are worth investigating from a developmental psychological perspective. Finally, the penal system as a space in which processes of change are to take place is analyzed from a developmental psychological perspective. It is argued that broadening the perspective on prisons in practice and research towards a developmental psychological angle has the potential to contribute to a broadening of the possibilities of promising interventions within prison.
\end{abstract}

Keywords Penal system · Rehabilitation - Treatment of offenders · Developmental psychology $\cdot$ Developmental regulation

\section{Strafhaft als Entwicklungsintervention}

Als vor gut 400 Jahren in Europa die ersten Zucht- und Arbeitshäuser Verbreitung fanden, verband sich die damals noch junge Idee vom Freiheitsentzug als strafrechtliche Sanktionsform für gewöhnliche Verbrecher bald mit dem Gedanken, die Zeit im Vollzug zu nutzen, um auf den Delinquenten einzuwirken und ihn zu ,bessern“ (Laubenthal 2019). Der Strafzweck der positiven Spezialprävention war geboren, wenngleich die Methoden zur Besserung zunächst wohl v.a. in Zucht und Erziehung zu Arbeit, Disziplin und Gottesfürchtigkeit gesehen wurden. Im Laufe des 19. und frühen 20. Jh. entwickelten mehrere Gefängnisreformbewegungen diesen Ansatz weiter und mit ihm zunehmend auch die Strafzwecktheorien. Der Erziehungsgedanke findet sich dabei noch heute explizit im Jugendgerichtsgesetz, in den gesetzlichen Bestimmungen für den Vollzug 
von Jugendstrafe ${ }^{1}$ und den Konzeptionen der Jugendstrafanstalten. Im Erwachsenenvollzug, der den Besserungsauftrag heute als Resozialisierungsauftrag auffasst, dürfte er zumindest implizit vielfach noch eine Rolle spielen - immerhin nannte das Schweizerische Strafgesetzbuch bis zum Jahr 2006 in Art. 37 die ,erzieherische Einwirkung“ auf den Gefangenen als einzige Methode zu seiner Resozialisierung (siehe hierzu Gautschie 1956). Der gemeinsame Nenner aller Konnotationen des Besserungsgedankens ist dabei die Intention, in die Entwicklung des Insassen einzugreifen, sie mindestens anders zu orientieren, und $u$. $U$. auch konkreter zu beeinflussen. Der Besserungsauftrag an den heutigen Strafvollzug (Maelicke und Suhling 2018) beinhaltet insoweit den Auftrag zur Veränderung ihrer Insassen in Richtung auf einen von außen gewünschten Zielzustand. Strafvollzug ist insoweit „Entwicklungsintervention“.

Als sich Ende der 1950er- und im Verlauf der 1960erJahre in Deutschland eine große Strafrechtsreform anbahnte, fanden die Planungen zu einer Zeit statt, in der Psychotherapie als Heilmethode psychischer Störungen zunehmend Akzeptanz und Verbreitung fand. Der Gedanke lag also nahe, (auch) therapeutische Behandlungsmethoden als Mittel zur Resozialisierung von Strafgefangenen einzusetzen. Die Idee des „Behandlungsvollzuges“ fand breiten Niederschlag in den Reformgesetzen der 1960er- und 1970er-Jahre; es entstanden sozialtherapeutische Anstalten. Aber auch im sog. Regelvollzug wurden und werden therapeutische Maßnahmen für Strafgefangene vorgehalten. Die Geschichte des behandlungsorientierten Strafvollzuges war seit ihren Anfängen wechselhaft, nicht zuletzt, weil Therapie ressourcen- und damit kostenintensiv ist und erste Evaluationen der Effekte enttäuschten (Lösel 1995). Seit gut zwei Dekaden hat Therapie im Strafvollzug aber einen steten Bedeutungszuwachs erfahren. Seither hat sich die Zahl sozialtherapeutischer Einrichtungen hierzulande mehr als verdreifacht ${ }^{2}$, der Kreis gesetzlich obligatorisch zu behandelnder Strafgefangener wurde stetig ausgeweitet, und auch sicherungsverwahrte Menschen haben mittlerweile einen gesetzlichen Anspruch ( $\$ 66 \mathrm{c} \mathrm{StGB}$ ) auf ein individuell zugeschnittenes Therapieangebot (Übersicht: Endres und Breuer 2018; Wischka und Boogaart 2018). ${ }^{3}$

Das Modell hat allerdings Grenzen. So kannte ja schon der Erziehungsvollzug Gefangene, die durch das erziehe-

\footnotetext{
1 Z.B. Niedersächsisches Justizvollzugsgesetz $\S 113$ (Vollzugsziele des Jugendvollzuges), Absatz 1 Satz 1: „Der Vollzug ist erzieherisch zu gestalten.".

2 Vgl. die jährliche Stichtagserhebung zur Sozialtherapie der Kriminologischen Zentralstelle Wiesbaden e. V.

3 Die gesetzlichen und anderen Reformen dieser Tendenz sind nicht ohne Ambivalenzen: So erzeugt etwa die Notwendigkeit, obligatorische Therapieplätze für bestimmte Gefangene vorzuhalten, neben positiven Anreizen z. B. auch Verdrängungseffekte, deren Kompensation schwierig ist.
}

rische Anliegen nicht erreicht wurden. Beispielsweise unterschied der Strafrechtstheoretiker Franz von Liszt (1905) zwischen den „besserungsfähigen und besserungswilligen“ (also die zu erziehenden), den „einschüchterungsfähigen“ (die keiner Erziehung bedürfen, sondern durch Abschreckung zu erreichen sind) und den „unverbesserlichen“ Verbrechern (die zu sichern sind). So wird das heute vermutlich niemand mehr bezeichnen wollen, obwohl die grundlegende Kategorisierung vielleicht nicht überall verschwunden ist. Jedenfalls finden sich auch im modernen Behandlungsvollzug Gefangene, die durch die verfügbaren Therapieangebote nicht erreicht werden, sich mitunter unter der Behandlung in ihrer Legalprognose gar verschlechtern. Es gibt Behandlungsabbrecher, deren Rückfallrisiko selbst gegenüber unbehandelten Gefangenen erhöht ist. Es gibt Ausgeschlossene mit bloß mittleren Rückfallrisiken, die durch die zunehmende Fixierung der Ressourcen des Behandlungsvollzugs auf Hochrisikogruppen entsprechend der „Risk-need-responsivity“(RNR)-Prinzipien wirksamer Straftäterbehandlung nicht mehr adressiert (oder erreicht) werden, und in den Behandlungseinrichtungen für Sicherungsverwahrte sammeln sich Gefangene mit jahrzehntelanger Vollzugserfahrung und vielfältig gescheiterten Behandlungsversuchen ohne realistische Entlassungsperspektive (Dahle 2019). Andererseits gibt es Gefangene, die auch ohne explizite therapeutische Behandlung im Vollzugsverlauf offenbar Veränderungen vollziehen und trotz erheblicher strafrechtlicher Vorgeschichte und ungünstiger Prognose nach Haftentlassung unerwartet einen legalen Lebensweg einschlagen (Bliesener 2003, 2014a). Strafvollzug wirkt offenbar vielfältig. Neben den oft untersuchten und vielfach replizierten intendierten Effekten (z.B. Lösel 2018, 2020; Lösel et al. 2019) gibt es demnach sowohl kontraintentionale als auch nonintentionale Wirkungen und Nebenwirkungen - erwünschte wie unerwünschte.

Der Umstand, dass die Intervention des Strafvollzugs nicht bei allen die intendierten Effekte erzielt, kann jedoch auch als Aufforderung gelesen werden, die sich im Ausbleiben angestrebter Wirkungen zeigende Erklärungslücke zu schließen und die Hintergründe und psychologischen Mechanismen dieser negativen wie auch unerwartet positiven Wirkungen des erziehungs- und behandlungsorientierten Strafvollzuges zu untersuchen (Suhling 2018; Suhling und Neumann 2015). Da es um die Ergründung der Rahmenbedingungen und Voraussetzungen für Veränderungsprozesse geht, bietet es sich an, hierbei entwicklungspsychologische Konzepte und Methoden heranzuziehen, weil die Entwicklungspsychologie als Wissenschaft von der menschlichen Entwicklung über die Lebensspanne Veränderungsprozesse und ihre Voraussetzungen zunächst allgemeiner adressiert, ohne von vornherein vom Anliegen eines von außen gedachten Zielzustandes auszugehen. Eine entwicklungspsychologische Perspektive wird einerseits 
die Prozesse thematisieren, die in einer angewandten pädagogischen (,Erziehungsvollzug“) oder klinischen (,Behandlungsvollzug") Perspektive als Grundlage für eine aussichtsreiche Intervention benötigt werden, dabei aber eine in praktischen Kontexten - und hier gerade auch im Strafvollzug - naheliegende instrumentelle Verkürzung (,what works“") vermeiden helfen, eben weil es ihr wesentlich um die Erklärung der zugrunde liegenden Prozesse geht (Ward 2020). In durch normative Zielsetzung stark bestimmten Kontexten wie dem Strafvollzug liegt zudem die Gefahr nahe, den Gefangenen tendenziell als Mangel- oder Defizitwesen zu begreifen (der ja gerade deshalb ,zu erziehen“ oder ,zu behandeln“ ist). Dies trägt zu einer einseitig risikofokussierten Sicht bei - möglicherweise auch in der Strafvollzugsforschung, die selbst da, wo sie Wirkungsforschung ist, zu selten an den wirksamen Entwicklungsprozessen ansetzt (Suhling 2018).

Der Grundgedanke des vorliegenden Aufsatzes ist es deshalb, dass es lohnenswert sein könnte, bei der Erforschung des Strafvollzuges mit seinem spezialpräventiven Besserungsauftrag den Fokus über bloße Erziehungs- und Behandlungseffekte hinaus zu öffnen und auf die grundlegenden Prozesse der und Rahmenbedingungen für Veränderungen dieser speziellen Klientel in dieser speziellen Umgebung zu erweitern - einschließlich der Veränderung dieser Prozesse. Gerade dann, wenn Entwicklungsverläufe und die sie regulierenden Prozesse interindividuell variieren, sollten die diese Differenzen erklärenden Prozesse von besonderem Interesse sein. So könnte das Interventionsziel einer Strafhaft ja gerade darin bestehen, nicht nur Verläufe zu verändern, sondern die sie steuernden Prozesse zu ermöglichen und die Voraussetzung für Entwicklung zu schaffen.

Dementsprechend könnten allgemeine entwicklungstheoretische Überlegungen den Rahmen eines auf den spezifischen Entwicklungskontext „Strafvollzug“ fokussierten Forschungsprogramms bilden. Dabei soll es nicht um eine spezielle Entwicklungspsychologie des Strafvollzuges gehen. Anliegen ist es vielmehr, allgemeine entwicklungspsychologische Konzepte, Prozessmodelle und Erkenntnisse (zusammenfassend etwa Ahnert 2014; Greve und Thomsen 2019a; Lerner 2002; Schneider und Lindenberger 2018) ebenso wie entwicklungsbezogene Ansätze der Kriminologie und Kriminalpsychologie (z.B. Day und Wiesner 2019; Farrington et al. 2019; Morizot und Kazemian 2015; Übersicht bei Dahle und Volbert 2005) für den speziellen Kontext des Strafvollzugs und seine spezielle Klientel heranzuziehen und auf ihre potentielle Nützlichkeit für die Ausweitung der bisherigen Grenzen des erziehungs- und behandlungsorientierten Strafvollzuges und ihre Verträglichkeit mit kriminalpsychologischen und kriminologischen, ggf. auch kriminaltherapeutischen Kon- zepten und Erkenntnissen, hin auszuloten. Es geht mithin um eine Entwicklungspsychologie im Strafvollzug.

\section{Grundzüge einer Entwicklungspsychologie im Strafvollzug}

Der folgende Abschnitt sichtet dementsprechend ausgewählte einschlägige Konzepte und Modelle der Entwicklungspsychologie der Lebensspanne mit dem Ziel, sie auf ihre Potenziale hin zu untersuchen, Veränderungsprozesse - oder ihr Ausbleiben - strafgefangener Menschen im Kontext einer Vollzugseinrichtung zu erklären (zur Anwendung auf deviante Entwicklungsverläufe: Day und Wiesner 2019). Leitend ist die von Baltes (1987), Brandtstädter (1990) und anderen vorgeschlagene Heuristik, dass die Humanontogenese (1) durch lebensaltersgebundene und insofern universelle Entwicklungssequenzen und -prozesse, (2) durch individuelle (,kritische“) Lebensereignisse und (3) durch kontextuelle (historische, kulturelle oder soziale) Bedingungen geformt wird. Für den Kontext dieser Überlegungen sind dabei insbesondere solche (ältere wie aktuelle) Überlegungen anschlussfähig, die Entwicklung als gerade auch durch Aufgaben und Herausforderungen beeinflusst und vorangetrieben beschreiben (zusammenfassend Baltes et al. 2006; Greve und Thomsen 2019a). Strafgefangene müssen sich, wie alle anderen Menschen, den universellen Aufgaben stellen, aber die auch durch besondere Lebenserfahrungen gekennzeichnete spezielle Klientel und der spezielle Vollzugskontext lassen für sie Besonderheiten erwarten. Der zweite Teil des Abschnitts widmet sich dann den in der aktuellen Entwicklungspsychologie des Erwachsenenalters zunehmend fokussierten Veränderungsund Regulationsprozessen und ihren Voraussetzungen und Einflussfaktoren. Wenn Entwicklung durch Prozesse gesteuert wird, die unter unterschiedlichen Randbedingungen unterschiedliche Entwicklungsfolgen produzieren, könnte hier ein interessanter und bislang $\mathrm{zu}$ wenig genutzter Ansatzpunkt für gezielte Interventionen liegen.

\section{Entwicklungsaufgaben}

Bedingt durch das strafrechtliche Eintrittsalter von 14 Jahren (in Deutschland) finden sich im Justizvollzug Menschen sehr unterschiedlichen Alters, in entsprechend unterschiedlichen Lebensphasen. Das Spektrum reicht von Jungtätern im gerade begonnenen Jugendalter bis zu Gefangenen im Seniorenalter, die sich nicht nur, aber insbesondere in den Einrichtungen zur Sicherungsverwahrung finden. Die Annahme liegt nahe, dass die Persönlichkeitsreife, das Selbstkonzept, aktuelle Lebensthemen, Ziele und Bedürfnisse, aber auch die Flexibilität, die Offenheit für neue Wege, die wahrgenommenen Ressourcen für Veränderungen und 
die Plastizität der Lebensorientierung (auch) altersbedingt höchst unterschiedlich sind. Als erster Ordnungsrahmen für eine entwicklungspsychologische Perspektive auf den Strafvollzug und ihre Gefangenen bietet sich deshalb eine Sequenzierung des Lebenslaufs auf der Grundlage der Systematik lebensphasenspezifischer Entwicklungsaufgaben an, die die Entwicklung im hier relevanten Jugend- und Erwachsenenalter anhand von biologisch, sozial (gesellschaftlich-kulturell) und individuell regulierten Entwicklungsaufgaben (Brandtstädter und Greve 2006; Havighurst 1972 [1948]) strukturiert. Dieser Ansatz typischerweise im Lebenszyklus auftretender (insoweit ,normativer“) Entwicklungsaufgaben wird im ersten Teil des Abschnitts angesprochen. Unvorhersehbare (nichtnormative) Entwicklungsaufgaben und -probleme, die die individuelle Ontogenese (z. B. den bisherigen Orientierungsrahmen eines Menschen) einschneidend verändern und insofern ,kritisch“ genannt werden können, werden im zweiten Teil angesprochen.

\section{Lebenszyklus und Entwicklungsaufgaben}

Dem Konzept normativer Entwicklungsaufgaben liegt die Idee einer mehr oder weniger prototypischen, teils biologisch, teils soziokulturell normierten Abfolge entwicklungsrelevanter Lebenskonstellationen (,Abschnitte“) zugrunde, in denen ein Mensch im Lauf seines Lebens in Abhängigkeit von gesellschaftlich und kulturell vermittelten Möglichkeiten und Erwartungen und von biologischen Notwendigkeiten mit lebensphasentypischen Problemen konfrontiert wird, die jeweils spezifische Anforderungen bereithalten. Dabei wird angenommen, dass es, jedenfalls innerhalb einer Generation, Zeitepoche und Kultur, ein zumindest partiell normativ vorgegebenes Grundmuster individueller Lebensläufe und der darin enthaltenen Abfolge bedeutsamer Entwicklungsaufgaben gibt (Freund und Baltes 2005). Dieses Grundmuster folgt demnach teilweise biologischen Notwendigkeiten (z.B. Generativität, Altersabbau körperlicher Funktionen, Begrenzung der Lebenszeit), v.a. aber auch sozial vermittelten Erwartungen (z. B. an Partnerschaft und Familie, Beruf, gesellschaftliche Rolle usw.), die den Rahmen vorgeben, innerhalb dessen sich individuelle Lebensläufe normalerweise entfalten. Die Grundhypothese ist, dass die Bearbeitung (,Bewältigung“) dieser Probleme Veränderungsprozesse und, jedenfalls für die Mehrzahl der Menschen, Entwicklung impliziert. In der Tradition von Havighurst (1972 [1948]) geht die kategoriale Ordnung von Aufgabentypen (bzw. -quellen) über die davor formulierten Sequenztheorien (insbesondere den Ansatz der Entwicklungskrisen von Erikson 1966 [1959], 1982) hinaus, eben weil neben die biologischen wesentlich auch sozial explizit oder implizit vorgegebene Entwicklungsaufgaben treten.

Diese normative und mindestens innerhalb eines soziokulturellen Kontextes daher universelle Sequenzierung wird mit dem beginnenden Erwachsenenalter freilich durch zunehmend selbstgesetzte (,autonome“) Entwicklungsaufgaben aufgebrochen und modifiziert. Im Anschluss an Überlegungen von Havighurst (1972 [1948]) hat dieser ,,aktionale“ Ansatz seit dem Ende des 20. Jh. an Bedeutung gewonnen (Baltes 1987; Brandtstädter 1990, 2001, 2006; Brandtstädter und Lerner 1999; Featherman und Lerner 1985; zur Anwendung auf kriminelle Lebensläufe: Day und Wiesner 2019). Entwicklung im Erwachsenenalter wird ohne Integration dieser Möglichkeit der Selbstgestaltung schwerlich erklärlich sein (Greve 2007; Greve und Thomsen 2019a) ${ }^{4}$. Gerade im Hinblick auf potenzielle Entwicklungsinterventionen eröffnet dieser Ansatz ein großes und wichtiges Spektrum an Einflussmöglichkeiten durch die Förderung von Kompetenzen und Orientierungen der Selbststeuerung.

Ein wichtiger Faktor der hier verfolgten Entwicklungsperspektive ist die offensichtliche Feststellung, dass es sich bei Strafgefangenen um Menschen handelt, die sich in ihren Handlungen wenigstens einmal - zumeist aber wiederholt weit außerhalb der gesellschaftlichen (normativen) Erwartungen bewegt haben. Dabei fußen einige Erklärungsmodelle kriminellen Verhaltens (z. B. Anomietheorien, u.a. Merton 1938) auf der Annahme, dass eine Ursache kriminellen Verhaltens eine Diskrepanz zwischen der Vermittlung gesellschaftlicher Erwartungen und der mangelnden Zurverfügungstellung der zu ihrer Erfüllung erforderlichen Ressourcen ist. Dies schafft für den Betroffenen die Notwendigkeit, Selbstkonzepte und persönliche Ziele zu entwickeln, die mit diesem Widerspruch umgehen, was wiederum die Wahrnehmung und den Einfluss eines gesellschaftlich vermittelten Skripts einer Normbiografie moderieren könnte. Es lassen sich bei strafgefangenen Menschen insofern Besonderheiten in den gesellschaftlich vermittelten Einflüssen auf die Wahrnehmung anstehender Entwicklungsaufgaben erwarten (Day und Wiesner 2019).

Zugleich findet Entwicklung immer im sozialen Kontext statt, der nicht nur Anforderungen stellt, sondern auch Ressourcen bereitstellt (oder vorenthält). Für den Strafvollzug ist dieser Kontext in aller Regel restriktiver und selektiver als in anderen Lebenswelten. Es kommt hinzu, dass sich der Justizvollzug in den vergangenen Dekaden zunehmend zu einem multikulturellen Schmelztiegel entwickelt hat, in dem Gefangene ganz unterschiedlicher Herkunft und Kultur in Zwangsgemeinschaft zusammenleben (Schmidt et al. 2016, 2019). Auch hier wird man also eine Varianz an kulturell vermittelten Vorstellungen von einem anzustrebenden Ideallebenslauf vermuten. Wenn man auf der anderen Sei-

\footnotetext{
${ }^{4}$ Allerdings können die für Selbstgestaltung und -regulation notwendigen Voraussetzungen in diesem Format nicht erklärt werden (Greve und Thomsen 2019b). Auf diesen Punkt wird an späterer Stelle noch einzugehen sein.
} 
te gleichwohl unterstellt, dass Abfolge und genaue Inhalte der antizipierten Entwicklungsaufgaben bei einer gefangenen Klientel zwar einerseits variieren und von der jeweils vorherrschenden idealtypischen Normbiografie mehr oder weniger abweichen mögen, andererseits aber die Notwendigkeit, das eigene Leben entlang bestimmter - normativer - Themen und Aufgaben zu choreografieren und zu strukturieren, auch bei strafgefangenen Menschen besteht, lassen sich die skizzierten Besonderheiten auch als Aufforderung verstehen, sie gerade in den Fokus von Forschung zu stellen. Immerhin zeigen empirische Befunde, dass wichtige Entwicklungsaufgaben durchaus auch Grundbedürfnisse strafgefangener Menschen treffen (z.B. nach Intimität und Partnerschaft; Barth 2015). Und tatsächlich lassen sich auch normative lebensabschnittabhängige Veränderungen bei der Klientel im Strafvollzug beobachten. Deutlich wird dies beispielsweise im Konzept der ,adolescence-limited offenders" im Zweipfadmodell von Moffitt (1993, 2006; McGee und Moffitt 2019), in dem das häufig beobachtbare Phänomen jugendtypischer Delinquenz, das sich mit Eintritt ins Erwachsenenalter von selbst erübrigt, mit hiermit einhergehenden veränderten Rollen, Aufgaben und Zielen erklärt wird - mithin mit der Zuwendung der Betroffenen zu den in der nächsten Lebensphase anstehenden Entwicklungsaufgaben. Es lohnt sich also, sich der Strukturierung der Lebensverläufe strafgefangener Menschen aus der Perspektive lebensphasenspezifischer Entwicklungsaufgaben anzunähern, gerade auch mit Blick auf mögliche Abweichungen vom idealtypisch-normativen Ablauf.

Die Annahme, dass sich auch der gesellschaftlich bedingte Möglichkeitsraum für Entwicklungsprozesse in Abhängigkeit vom Alter verändern könnte, dass biologische, soziostrukturelle und kulturell vermittelte Einflüsse die Ressourcen zur Bewältigung von Entwicklungsaufgaben in Abhängigkeit von der Lebensphase moderieren könnten und altersabhängige Zeitfenster vorgeben, in denen nicht nur die Beschäftigung mit bestimmten Lebensthemen (eben Entwicklungsaufgaben) erwartet werden, sondern auch die gesellschaftlich vermittelten Ressourcen zu ihrer Bewältigung zur Verfügung gestellt werden, spitzen diesen Punkt zu. Demnach ist, mit Ausnahme biologischer Grenzen (z.B. im Hinblick auf eigene Kinder), das Ignorieren von „developmental deadlines“ (Heckhausen et al. 2019; Wrosch und Heckhausen 2005) zwar möglich, ein verspäteter Bewältigungsversuch aber mit ungleich höheren Kosten und zunehmend reduzierten Ressourcen verbunden. Diese Rahmenbedingung würde insoweit gerade auch nonnormative Lebensverläufe betreffen: Das Verpassen des normativen (günstigen) Zeitfensters ginge mit zunehmend reduzierten Chancen auf eine individuell befriedigende Bewältigung von Entwicklungsaufgaben einher. Zu beachten ist im hiesigen Kontext, dass sich im Justizvollzug nicht wenige Langzeitgefangene befinden, die diese optimalen Zeitfenster für altersgemäße Entwicklungsprozesse entlang biografisch sequenzierter Themen unter den einschränkenden Bedingungen einer Haftanstalt erleben, die weder die sonst erwartbaren Handlungs- und Entwicklungsoptionen noch die erforderlichen Bewältigungsressourcen zur Verfügung stellt. Die Wahrscheinlichkeit, im Verlauf einer langen Freiheitsstrafe Entwicklungsaufgaben nicht oder nur unzureichend befriedigend zu bewältigen und bei Haftentlassung dann das passende Zeitfenster verpasst zu haben, scheint aus dieser Perspektive nicht gering. Die hiervon Betroffenen würden sich insoweit der Aufgabe gegenübersehen, entweder mit sehr hohem - vielleicht unzureichend möglichem - Ressourceneinsatz verpasste Entwicklungschancen nachzuholen oder Bedürfnisse und Lebensziele an die nunmehrige Realität anzupassen. Es kommt hinzu, dass im Verlauf einer Strafhaft kontextspezifische (,intramurale“) Entwicklungsaufgaben zu lösen sind, wie z.B. die gleichzeitige Anpassung an vollzugliche Strukturen wie auch an spezifische (Sub-)Kulturen und ihre Rahmenbedingungen. Die Bewältigung dieser Aufgaben wird nur bedingt nützlich für die weitere Entwicklung nach der Haftentlassung sein, ist aber für die Zeit der Inhaftierung unabweislich - drängender womöglich als die eigentlich ,altersgemäßen“ Entwicklungsaufgaben.

Die Idee der Strukturierung des Lebenslaufs anhand normativer Sequenzen von Entwicklungsaufgaben ist nicht ohne Kritik geblieben. Bemängelt wurden insbesondere ihr universeller Anspruch und ihr unidirektionaler Charakter, der etwa in der Konzipierung nach Erickson Merkmale eines klassischen Stufenmodells aufweist. Indessen ist zumindest Unidirektionalität nicht zwingend ein notwendiges Element des Ansatzes von Entwicklungsaufgaben. So weist beispielsweise das Konzept des Lebenszyklus nach Levinson $(1978,1986)$ auf die Möglichkeit eines bedingt-zirkulären Phasenverlaufs hin, demnach der initiale Entwurf einer ersten Lebensstruktur in der Adoleszenz durchaus um das 30. Lebensjahr (demnach in der "Transitionsphase“ ins mittlere Lebensalter) herum noch umfassenderen Korrekturen zugänglich sein soll. Eine solche Variante der nachträglichen umfassenden Korrektur eines ersten Lebensentwurfs ließ sich dabei im Rahmen der Berliner CRIMELängsschnittstudie beobachten. Hier war es einer Gruppe von immerhin $11 \%$ der 307 Probanden der Studie gelungen, nach einer intensiven delinquenten Jugend und einer im Jungerwachsenenalter noch einmal deutlich gesteigerten kriminellen Phase in diesem Lebensabschnitt - und nach längerer Freiheitsstrafe - unvermittelt den bis dato eingeschlagenen delinquenten Lebenspfad zu verlassen und im Weiteren ein legales Leben zu führen (,,altersbegrenzte Intensivtäter“; Bliesener 2009, 2010, 2012; Dahle 1998, 2001, 2005a; Stelly und Thomas 2005; Taefi und Hosser 2019).

Trotz der im Grundsatz wohl berechtigten Kritik am weitreichenden Anspruch genereller Sequenzmodelle (zusammenfassend Greve und Thomsen 2019a) und der oben 
begründeten Vermutung, dass bei der relevanten Zielgruppe weitere Besonderheiten zu erwarten sind, scheint der Ansatz für das hiesige Anliegen vielversprechend. Immerhin ist mit Blick auf die Anomietheorie der Gedanke ja nicht abwegig, dass nichtwenige strafgefangene Menschen durchaus bürgerliche Vorstellungen von einem idealen Lebenslauf mit weiten Teilen der Gesellschaft teilen könnten, jedoch die Mittel und Möglichkeiten zur Umsetzung fehlen. Dies aber würde bedeuten, dass dieselben Entwicklungsaufgaben, die sich den Menschen einer Kohorte stellen, durchaus auch im Laufe des Lebens von Straftätern Relevanz haben, Art und Möglichkeiten ihrer Lösung jedoch eingeschränkt, zumindest divergent sind. Diese Vermutung legt auch das „good lives model of offender rehabilitation“ nahe (GLM; Ward und Gannon 2006). Im Kern geht das GLM von einer erschöpfenden Anzahl universeller menschlicher Bedürfnisse (,primary goods“) aus, die Rechtsbrecher mit anderen Menschen teilen. Kriminelles Verhalten entsteht, so die Annahme, wenn dem Einzelnen die internen und externen Ressourcen (,secondary goods“) fehlen, um diese Bedürfnisse mit prosozialen Mitteln zu befriedigen, oder wenn einzelne Bedürfnisse zulasten der übrigen überbewertet werden und ein Ungleichgewicht entsteht.

\section{Kritische Ereignisse als nonnormative Entwicklungsaufgabe}

Die Perspektive auf Entwicklung als einen durch mehr oder weniger normierte (bzw. gewählte) Entwicklungsaufgaben strukturierten und vorangetriebenen Prozess wird durch das Konzept der „kritischen Lebensereignisse“ (Filipp und Aymanns 2010) ergänzt. Gemeint sind plötzliche Einschnitte in das Leben des Menschen durch gravierende Ereignisse, die individuell als negativ („Krise“) oder positiv (,Chance“) erlebt werden. Im extremeren Fall ändern sich nicht nur die Randbedingungen für die zukünftige Entwicklung, sondern auch die Regeln, nach denen das Leben des Betroffenen bislang organisiert war. Sie erfordern insoweit eine Anpassung der bisherigen Orientierungen und Lebenspläne an den neuen Kontext. Auch zu ihrer Bewältigung werden interne und externe Ressourcen benötigt.

Das Konzept der kritischen Lebensereignisse findet seine kriminalpsychologische Entsprechung beispielsweise im Modell der ,age-graded theory of informal social control“" (Laub und Sampson 2001), demnach es auch im Leben eines chronischen Rechtsbrechers mit verfestigtem dissozialen Lebensstil „turning points“ geben kann, die unerwartete Veränderungen in grundlegenden Rahmenbedingungen der bisherigen Lebensführung mit sich bringen (z. B. eine neue Partnerschaft, plötzliche Pflegebedürftigkeit eines nahen Angehörigen oder eine unerwartete berufliche Chance; Day und Wiesner 2019). Sie gehen mit veränderten Lebensroutinen und einer veränderten Bilanz sozialen Kapitals einher, die dazu führen, dass sich ein Straftäter umorientieren und auch nach verfestigter krimineller Karriere einen veränderten Lebenspfad einschlagen kann (Taefi und Hosser 2019). Diese nonnormativen Ereignisse sind dem Modell nach weder plan- noch willkürlich herstellbar.

Interessant werden kritische Lebensereignisse im hiesigen Kontext zusätzlich dadurch, dass auch die Tatsache des Strafvollzugs selbst (insbesondere als Erstinhaftierter oder als Verurteilter mit drohender oder angeordneter Sicherungsverwahrung) ein derartiges kritisches Lebensereignis, das den bisherigen Orientierungsrahmen des Betroffenen aufbricht, darstellen oder hierzu zumindest beitragen könnte (Hosser und Greve 2005; Kazemian und Walker 2019). Nicht umsonst kennt die Strafvollzugspsychologie den Begriff des „Inhaftierungsschocks“, der u.a. für die Tatsache eines erhöhten Suizidrisikos insbesondere in den ersten Hafttagen verantwortlich gemacht wird (Dahle et al. 2005a), der sich aber generell in einer insbesondere zu Haftbeginn relativ höheren Belastung zeigt (z.B. Greve et al. 2001; Greve und Enzmann 2003). Aber auch der weitere Vollzug birgt erhöhte Risiken für Krisen, die durch kritische Ereignisse im Verein mit eingeschränkten Reaktionsmöglichkeiten ausgelöst werden. Anlässe können etwa Trennungen von Partnern sein, der Tod naher Angehöriger oder auch die letztendliche Anordnung von Sicherungsverwahrung nach jahrelanger Hoffnung, die Maßregel vermeiden zu können. Sie erfordern in der Folge nicht selten eine Krisenintervention durch Vollzugspsychologen oder -psychiater (Mechler 1981). Aber auch die Erfahrung einer gravierenden Straftat selbst kann ein derartiges kritisches Ereignis darstellen, wenn die Tat dem bisherigen Selbstkonzept des Täters fundamental widerspricht.

Wenn man also annimmt, dass das Gefängnis das Risiko kritischer Lebensereignisse erhöht und die Insassen ihrerseits erhöhte Risiken kritischer Lebensereignisse mitbringen, erscheint ein Blick in den Vollzug unter dem Blickwinkel auch solcher Entwicklungsaufgaben vielversprechend. Zwar sind systembedingt die Risiken v. a. für negativ konnotierte Ereignisse erhöht. Wie jede Krise bergen jedoch auch sie Entwicklungschancen.

\section{Zwischenfazit}

Führt man abschließend die Grundgedanken normativer und nonnormativer Entwicklungsaufgaben zusammen, so fällt eine besondere Aufgabe ins Auge, die sich einem Gefangenen jedenfalls dann stellt, wenn er sich dem Gedanken nähert, einen bis dato intensiven kriminellen Lebenspfad zu verlassen. Demnach ist ja eine der zentralen Entwicklungsaufgaben, die sich dem Menschen auf dem Weg ins Erwachsenenalter früher oder später stellt, der Entwurf eines Lebenskonzepts, das Orientierung bietet, den weiteren Lebensweg strukturiert und dem Betroffenen einen Platz in der Gesellschaft zuweist. In der Systematik nach Levinson 
(1978, 1986) ist dies das zentrale Thema des Jungerwachsenenalters (,early adulthood“), wobei er, wie schon dargelegt, in der Übergangsphase ins mittlere Erwachsenenalter noch einmal die Möglichkeit für auch umfassendere Korrekturen sieht. Man erhält in seiner Systematik gewissermaßen eine zweite Chance, auch grobe Irrtümer zu heilen. Dem lässt sich entnehmen, dass der erste Entwurf noch eine gewisse Plastizität aufweist, die Offenheit und Fähigkeit für einen Neuanfang in der Transitionsphase ins mittlere Erwachsenenalter noch gegeben ist, er andererseits solche umfassenderen Korrekturen in späteren Lebensabschnitten aber als offenbar zunehmend schwierig ansieht. Die Plastizität des Lebensentwurfs nimmt demnach in den folgenden Lebensabschnitten ab, was in Anbetracht der Bedeutung des Lebenskonzepts für das Selbstbild und die Selbstwahrnehmung vielleicht nicht ganz ohne Plausibilität ist und durch das bereits erwähnte Konzept der ,developmental deadlines" unterstrichen wird.

Folgt man dem, so wäre also auch das Verlassen eines kriminellen Lebensentwurfs für Strafgefangene mit zunehmendem Alter eine zunehmend schwierige Aufgabe. Tatsächlich kennt die kriminologische „Desistance“-Theorie solche Hemmnisse, die möglicherweise mit dem Alter eine zunehmende Hürde darstellen könnten. So bedeutet in der Lesart der Theorien der kognitiven Transformation (z. B. Giordano et al. 2002; Maruna 2001) Desistance, also ein Ausstieg aus einer intensiven kriminellen Karriere, v. a. eine Transformation des Selbstkonzeptes. Die Aufgabe besteht für den Betreffenden dabei darin, in selbstwerterhaltender Weise ein Narrativ zu entwickeln, das es ihm erlaubt, seine Vergangenheit als krimineller Mensch kohärent in eine neue Identität als rechtschaffender Bürger zu überführen. Dies stellt für einen Sicherungsverwahrten an der Schwelle zur siebten Lebensdekade und mit mehr als der Hälfte des Lebens Hafterfahrung vermutlich eine ungleich schwierigere Aufgabe dar als für einen jungen Intensivtäter im Alter von 30 Jahren oder für einen jugendlichen Gewalttäter. Das führt zu der Hypothese, dass mit zunehmender Hafterfahrung und zunehmendem Alter in Anbetracht abnehmender Restlebenszeit der Veränderungsdruck bei manchem Langzeitstraftäter zwar steigen könnte. Gleichzeitig wachsen aber die Anforderungen, die eine solche Veränderung an die Anpassung des Selbstkonzepts stellt, und auch die Ressourcen und realen Möglichkeiten für einen legalen Lebenswandel dürften mit zunehmend chronifizierter Straftäterkarriere und zunehmendem Lebensalter abnehmen. Insofern wird sich vermutlich der Druck erhöhen, die im bisherigen Lebensentwurf enthaltenen Ziele und Ansprüche an reale Gegebenheiten anzupassen. Plastisch wird dieser Druck beispielsweise bei denjenigen langjährig haftsozialisierten Langzeitgefangenen und Sicherungsverwahrten, deren Entlassungsperspektive nach Jahrzehnten der Haft in be- treuten und kontrollierten Wohnverhältnissen besteht (Dahle 2019).

\section{Entwicklungsregulative Prozesse}

Folgt man der Feststellung, dass einerseits normative Entwicklungsziele und Aufgaben, andererseits nonnormative Herausforderungen und der spezifische Kontext des Strafvollzugs die Aufgabenkonstellation ausmachen, denen sich Strafgefangene in diesem besonderen Entwicklungskontext stellen müssen, schließt sich die Frage nach den personalen und sozialen Faktoren und Ressourcen an, die die individuelle Auswahl von Entwicklungszielen und deren Zielverfolgung fördern oder behindern. Im Zentrum stehen hier Prozesse der individuellen Selbstregulation, deren Veränderlichkeit im Haftverlauf und diesbezügliche interpersonelle Unterschiede.

Im Falle der bereits angesprochenen ,persistent offenders“, die für einen Großteil der gravierenderen Straftaten verantwortlich sind (Moffitt 1993, 2006; Bliesener 2012, 2014b), ist beispielsweise die im interindividuellen Vergleich hohe und stabile Bereitschaft, bestimmte Ziele zu verfolgen und hierfür bestimmte und auch unerlaubte Mittel einzusetzen, eine naheliegende Erklärungsoption für ihre „Persistenz“ (sofern nicht die dauerhaft fehlende Selbstregulation die Ursache für die kontinuierliche Antisozialität ist). Eine differenzielle Perspektive würde, auch wenn sie über diese sehr globale Zweiwegetypologie hinaus differenziert, hier zwar die Möglichkeit einer nomothetischen (Kriminal-)Prognose eröffnen (,Die Wahrscheinlichkeit delinquenten Handelns ist für Person(typ) A mit den Merkmalen X höher als für Person(typ) B ohne diese Merkmale“), bietet aber zunächst noch keine Erklärung für das gezeigte Verhalten (Ward 2020) und kann auch die z.T. große Variabilität der Entwicklungsverläufe innerhalb der je spezifizierten Gruppe nicht aufklären. Die Frage nach den Ursachen und Hintergründen interindividueller Unterschiede als Erklärung (,Warum hat er das getan (und jener nicht)?“) führt insofern zur ontogenetischen Perspektive: Welche Entwicklungsbedingungen haben dazu geführt, dass diese Person genau diese Ziele und Handlungsoptionen gesehen und verfolgt hat? Dieses Anliegen deckt sich mit dem idiografischen methodischen Ansatz der (Kriminal-)Prognose, deren Kern aus der Begründung einer spezifischen, auf den Einzelfall zugeschnittenen individuellen Kriminaltheorie (,Delinquenzhypothese“) auf der Grundlage hermeneutischer Rekonstruktionen der biografischen Entwicklung (Dahle 1998, 2005b, 2010, Dahle und Lehmann 2018) besteht.

Die Chancen einer Zusammenführung der differenziellen und idiografischen Perspektiven mit einem entwicklungspsychologischen Ansatz erscheinen auch deswegen vielversprechend, weil die spezifischen Entwicklungsbe- 
sonderheiten einer straffälligen, zumal inhaftierten Klientel in den vorliegenden Kriminalitätstheorien bislang primär unter dem defizitorientierten Blickwinkel widriger Sozialisationseinflüsse und Entwicklungsbedingungen betrachtet wurden (z. B. im Modell kumulativer Risikofaktoren; u.a. Lösel und Bliesener 2003), seltener auch unter dem Aspekt kompensatorischer Schutzfaktoren (Bliesener 2003, 2014b, 2017; Lösel und Bliesener 1998). Dies steht in der oben angedeuteten Tradition einer defizit- und risikofokussierten Herangehensweise, die letztlich nach personalen Risikomerkmalen sucht, von deren (therapieinduzierten) Veränderung man sich im Sinne des RNR-Modells (Bonta und Andrews 2017) und der ,What-works“-Debatte effizienter Straftäterbehandlung (z.B. Weisburd et al. 2016) eine Reduktion von Rückfallrisiken verspricht. Die eigentlichen Entwicklungsprozesse hierbei waren bislang hingegen selten Gegenstand.

Die Notwendigkeit, eine biografisch-ontogenetische Perspektive mit der interindividuellen Variabilität von Entwicklungsverläufen von Straftätern zu verbinden, legt es jedoch nahe, den Blick weniger auf individuelle Eigenschaften bzw. Persönlichkeitsmerkmale als vielmehr auf entwicklungsregulative Prozesse zu richten. Hierbei stehen handlungssteuernde Aspekte der Person im Vordergrund. Diese umfassen auf personaler Ebene v. a. Intentionen und Motivation, aber auch deren Komponenten und Bedingungen wie etwa subjektive Theorien, Ziele und Erwartungen, dann aber auch subpersonale Ressourcen wie insbesondere Bewältigungsfähigkeiten sowie affektive und emotionsregulative Prozesse.

\section{Die Person als Akteur der eigenen Entwicklung: die aktionale Entwicklungsperspektive}

Mit der Perspektivenausweitung der Entwicklungspsychologie auf das Erwachsenenalter (Brandtstädter und Lindenberger 2007) ging, wie oben angesprochen, die bei Havighurst bereits angelegte, aber wenig ausgearbeitete Öffnung von Entwicklungstheorien für eine ,aktionale Perspektive“ (Brandtstädter 2001, 2006; Brandtstädter und Greve 2006; Brandtstädter und Lerner 1999; Leipold 2020) einher, die die Spielräume der Selbstgestaltung sichtbar machen, die ab dem frühen Jugendalter zunehmend entwicklungsrelevant werden. Entwicklung wird danach als partielle Selbstgestaltung gesehen, allerdings im Rahmen der je gegebenen Spielräume bzw. des „Möglichkeitskorridors“. Dieser wird nicht nur durch soziale und kulturelle Vorgaben begrenzt, sondern auch durch die jeweils individuellen Plastizitätsgrenzen. Man kann sich zu einem gegebenen Zeitpunkt der Biografie nicht beliebig - und gewiss nicht beliebig schnell - verändern. Nicht zuletzt gilt dies unter den besonderen institutionellen Rahmenbedingungen des Strafvollzugs, der vielfältige und auch widersprüchliche Anforderungen an zu leistende Anpassungsprozesse stellt und den Möglichkeitskorridor damit nicht unerheblich definiert.

Richtet man vor diesem Hintergrund den Blick auf den Prozess der Selbstregulation und aktionalen Selbstentwicklung, stellt sich zunächst die Frage, inwieweit Personen generell und Inhaftierte im Besonderen motiviert und befähigt werden können, durch das Setzen und bewusste Verfolgen von Zielen die eigene Entwicklung systematisch zu beeinflussen (Greve und Thomsen 2019a, b; Leipold 2020) und sich auf Basis der eigenen Lernerfahrungen im Sinne einer sozial verantwortlichen Selbstverwirklichung zu entwickeln. Eine wesentliche Voraussetzung für das Gelingen solcher Formen von Zielentwicklung stellen dabei Regulationsprozesse im Sinne von Bewältigungs- und Emotionsregulationsstrategien dar.

Die Unterscheidung zwischen ,personalen“, d.h. durch die Person jedenfalls prinzipiell bewusst steuerbaren Aktivitäten (z.B. der Wahl eines Ausbildungsweges oder die Entscheidung, sich im Vollzug für eine Sozialtherapie zu bewerben), und „subpersonalen“ Prozessen, d.h. Prozessen, die innerhalb der Person stattfinden (z. B. die Wahrnehmung einer sozialen Äußerung als „Provokation“ und die emotionale Reaktion darauf), ist gerade aus einer aktionalen Perspektive folgenreich. „Handlung“ ist als personales Konzept (Personen handeln; Neuronen handeln nicht) von subpersonalen Prozessen der Zielregulation konzeptuell zu unterscheiden. Beispielsweise erfolgen Präferenzanpassungen, wie etwa die mit dem Alter nachlassende Attraktivität von Gewalt, ohne dass diese Veränderung je Gegenstand einer bewussten Entscheidung gewesen ist. Gleichzeitig sind aber zahlreiche subpersonale Prozesse (etwa der Informationsverarbeitung oder der Emotionsregulation) notwendige (konstitutive) Bedingung für Handlungen. Die Förderung der aktionalen Selbstgestaltung setzt daher gerade im Strafvollzug - bei einer in der Selbstregulation typischerweise defizitären Klientel (Day und Wiesner 2019) - die Beachtung beider Ebenen der Selbstregulation voraus. Es ist daher lohnenswert, insbesondere die subpersonalen Prozesse näher zu betrachten, zumal sie in den vorliegenden Ansätzen zur Entwicklung von Straftätern (im Vollzug) bislang weniger beachtet worden sind.

\section{Prozesse der Zielentwicklung}

Auf dieser Betrachtungsebene sind im Hinblick auf das Vollzugsziel der Resozialisierung insbesondere solche Handlungsintentionen und Motivstrukturen von Interesse, die der sozialen Integration und Rückfallvermeidung dienen. Tatsächlich sind Motivstrukturen Kernbestandteil zahlreicher kriminologischer und kriminalpsychologischer Erklärungsansätze (zusammenfassend Suhling und Greve 2010). Zu nennen wären etwa „Rational Choice“-Theorien, die delinquentes Verhalten als Ergebnis einer in gewisser 
Hinsicht rationalen Nützlichkeitsabwägung zwischen den Vorteilen einer illegalen Handlung und dem Entdeckungsrisiko und den damit verbundenen Kosten zu erklären suchen (zur Kritik: Karstedt und Greve 1996). Sie finden sich aber auch in komplexeren Modellen, die, wie z.B. die ,situational action theory“ (SAT; Wikström 2014) oder dem Modell der ,psychology of criminal conduct" (PCC; Bonta und Andrews 2017), eine situationsunabhängige Entscheidungsneigung für delinquente Handlungsalternativen in einen breiteren Kontext sozialer und sozialisierter Faktoren einzubetten suchen.

Die Attraktivität bestimmter Ziele und die subjektive und objektive Verfügbarkeit und Wählbarkeit bestimmter Mittel wird dabei nicht nur vom aktuellen Kontext (,Gelegenheiten“), sondern wesentlich vom subjektiven und objektiven Möglichkeitsraum bestimmt, der die Handlungsauswahl der in einem spezifischen (sub-)kulturellen Kontext handelnden Personen kanalisiert. Dabei wird nicht nur die Attraktivität bestimmter Zielzustände (materielle Sicherheit, Autonomie usf.) kulturell vermittelt. Beispielsweise ist, wie angesprochen, der zentrale Punkt der Anomietheorie nach Merton (1938) die Überlegung, dass die Ressourcen, mit denen diese Ziele erreicht werden können, ungleich verteilt sind. Bestimmten Personengruppen bleibt demnach die Erreichung der allgemein als anzustreben definierten Ziele auf legalem Wege versperrt, weswegen illegale Mittel an Bedeutung gewinnen.

In wichtiger Hinsicht geht es also darum, Straftäter zunehmend in die Lage zu versetzen, ihre bisherigen negativen und positiven Erfahrungen in ihr Selbstbild zu integrieren, aus Fehlern zu lernen und sich vor dem Hintergrund dieser gesammelten Lernerfahrungen künftig auch selbstbestimmt sozial konforme Ziele zu setzen und zu verfolgen. Das Erleben von Konsistenz zwischen Selbstkonzept und Handeln trägt dazu bei, dass die Person zunehmend zu einem aktiven und bewussten Mitgestalter ihrer eigenen Entwicklung wird, mit Lebenszufriedenheit sowie Leistungsfähigkeit und Leidensfähigkeit (Kuhl und Hofmann 2019), der Fähigkeit, realistische Vorsätze in die Wirklichkeit umzusetzen und widrige Umstände unter Verzicht auf dysfunktionale Abwehrmechanismen oder Neutralisierungsstrategien auszuhalten und daran zu wachsen.

Aber selbst wenn allgemeingültige und/oder legitime spezifische Ziele von der Person definiert und festgelegt wurden und geeignete Mittel zur Zielerreichung prinzipiell verfügbar sein sollten, heißt dies nicht unbedingt, dass die Absichten damit ungehindert in entsprechende Handlungen umgesetzt werden. Hierzu bedarf es, gerade wenn dafür Verhaltensweisen erforderlich sind, die jenseits der üblichen Verhaltensroutinen liegen, auch der Fähigkeit zur Verhaltenssteuerung (Grieger et al. 2012). Eine kompetente Selbstregulation liegt dann vor, wenn das Verhalten entweder durch die eigenen Intentionen gesteuert werden kann oder mindestens ihnen entsprechend verläuft, wobei grundsätzlich eine eher automatisierte (,,intuitive“) Verhaltenssteuerung von einer eher bewussten ,aktionalen“ Steuerung zu differenzieren ist (Chaiken und Trope 1999). Darüber hinaus sind auch in den stärker reflexiv (bewusst gesteuerten) Handlungen im engeren Sinne verschiedene Steuerungsschritte zu unterscheiden. Allerdings sind hier die in der Motivationspsychologie bereits seit Längerem etablierten volitionalen Komponenten der Realisierung von Intentionen (,Warum hat er das getan - und nicht gelassen?") in kriminologischen Handlungstheorien bislang wenig ausgearbeitet worden. Auch dieser Aspekt der Kriminalitätserklärung verdiente mehr Aufmerksamkeit; jedoch liegen hierzu bislang aus einer Entwicklungsperspektive nur wenig Vorarbeiten vor (zu einer Ausnahme: Heckhausen et al. 2010, 2019), vielleicht auch deswegen, weil die relevanten Prozesse (etwa der Aufmerksamkeitssteuerung) ihrerseits überwiegend automatisch sind (zusammenfassend Suhling und Greve 2010).

Wenn die aktionale Perspektive der Entwicklungspsychologie für die Entwicklung im Vollzugskontext genutzt werden soll, ist es daher im ersten Schritt vielleicht aussichtsreicher, die subpersonalen Prozesse der Intentionsentwicklung, insbesondere die Zielauswahl und -anpassung stärker in den Blick zu nehmen, zumal hierzu mehrere Ansätze der Entwicklungspsychologie vorliegen. Diese Ansätze werden in der vollzuglichen Intervention bislang kaum genutzt. Ein wichtiger Ausgangspunkt ist die Überlegung, dass die aktionale Perspektive generell und insbesondere in Bezug auf Selbstregulation prinzipielle Grenzen hat. Über den oben bereits angesprochenen Punkt hinaus, dass unserer Selbstgestaltung äußere (physische, soziale, im Vollzugskontext insbesondere auch institutionelle) und innere Grenzen gesetzt sind (wir können nicht alles aus uns machen, gewiss nicht jederzeit - und manches realistisch gar nicht), ist v. a. die Entwicklung der intraindividuellen Handlungsbedingungen nicht in handlungstheoretischer Form $\mathrm{zu}$ rekonstruieren, wenn ein infiniter Regress vermieden werden soll. So können die Entstehung und Veränderung von Handlungszielen nicht selbst Handlungsziele sein; spätestens die Erklärung dieses Handlungszieles kann deshalb nicht mehr auf ein Handlungsziel verweisen. Also muss die Zielentwicklung durch andere (Entwicklungs-)Regulationsprozesse erklärt werden, die eben nicht mehr in einem aktionalen Format konzipiert sein dürfen (Brandtstädter 2001, 2006, 2007).

\section{Zielentwicklung: Prozesse und Bedingungen der Entwicklungsregulation}

Typische Ansätze entwicklungspsychologischer Regulationstheorien sind insbesondere das SOK(Selektion, Optimierung und Kompensation)-Modell (Baltes und Baltes 
1990; Freund 2008; Freund et al. 1999), die Kontrolltheorie der Lebensspanne (Heckhausen et al. 2010, 2019) sowie das Zweiprozessmodell der Entwicklungsregulation (Brandtstädter 2006, 2007, 2015; Brandtstädter und Rothermund 2002). Ihr gemeinsamer Fokus ist die Untersuchung der Prozesse, die einerseits die Verfolgung von (Entwicklungs-)Zielen ermöglichen und andererseits den Umgang mit Hindernissen, Grenzen oder Widerständen regeln (zusammenfassend: Greve und Leipold 2018). Bei allen Unterschieden in den Details ihrer Akzentuierung (Boerner und Jopp 2007; Haase et al. 2013) geht die gemeinsame Argumentationslinie von einem aktionalen Entwicklungsverständnis aus: Sowohl das SOK-Modell als auch die Kontrolltheorie der Lebensspanne betrachten Entwicklungsregulation dabei in wesentlicher Hinsicht als strategisches Geschehen, obwohl die bewusste Absichtlichkeit der Reaktionen nicht immer behauptet wird. Durch systematische Selektion der (noch) erreichbaren Ziele, durch hartnäckige Optimierung der eigenen für ihre Erreichung verfügbaren Strategien und durch Kompensation der gleichwohl fehlenden Mittel (Baltes und Baltes 1990; Freund 2008) können, so die Annahme, fehlende Möglichkeiten oder Zielblockaden häufig auch unter widrigen Lebenskonstellationen aufgefangen werden. Diese Modelle erläutern die oben als ,aktional“ gekennzeichnete Perspektive.

Für den Umgang mit den Grenzen der Steuer- und Kontrollierbarkeit hat die Kontrolltheorie der Lebensspanne vorgeschlagen, dass sich insbesondere in der Konfrontation mit als unüberwindbar erlebten Hindernissen die Person selbst verändern müsse (Heckhausen et al. 2010, 2019); beispielsweise dadurch, dass erreichbare Ziele gewählt werden. Übersetzt in den hier relevanten Kontext, impliziert dies, dass sich beispielsweise einige im Langzeitvollzug gealterte Gefangene sehr wahrscheinlich von zentralen früheren Lebenszielen lösen und in realistische Ziele transformieren müssten, wenn sie eine realistische extramurale Perspektive gewinnen wollen. Allerdings lassen sich, wie angesprochen, die Wahl und die Veränderung von Zielen und Absichten nicht intentional rekonstruieren: Wir können offenbar auch Ziele, die wir selbst zwar als hinderlich, belastend und unerreichbar erleben, nicht ohne Weiteres absichtlich degradieren oder aufgeben und ersetzen. So basieren beispielsweise Trauerreaktionen bei unabwendbaren Verlusten ja gerade darauf, dass dies nicht unmittelbar gelingt. Offenbar müssen Prozesse, die mit aktuell oder grundsätzlich nichtkontrollierbaren Schwierigkeiten umzugehen helfen, anders erklärt werden. Überdies wird in den primär einer aktionalen Perspektive verhafteten Modellen nicht systematisch geklärt, wie selektierende, optimierende und kompensierende Prozesse ihrerseits erklärt werden können.
Das Zweiprozessmodell der Entwicklungsregulation (Brandtstädter 2015; Brandtstädter und Rothermund 2002) hat hier - neben einem ,assimilativen“ Modus, der sich in vielem mit den SOK-Strategien bzw. ,primärer“ Kontrolle überschneidet - einen adaptiven (,akkommodativen“) Modus postuliert, in dem Ziele, Wünsche, normative Orientierungen und Präferenzen den wahrgenommenen Gegebenheiten so angepasst werden, dass Unerreichbares abgewertet oder durch Erreichbares ersetzt wird (zusammenfassend Greve und Leipold 2018). Während assimilative Regulationen (der hartnäckigen Zielverfolgung und Mittelanpassung) typischerweise ,personal“ (intentional, z. B. strategisch) gesteuert sind, sind akkommodative Regulationsprozesse (die nachlassende Wichtigkeit unerreichbarer Ziele) typischerweise subpersonal. Aktuell ist die Frage, wie diese akkommodativen Prozesse ihrerseits gesteuert werden, auch in diesem Modell nicht detailliert geklärt (Brandtstädter und Rothermund 2002 für eine diesbezügliche Heuristik), aber sie arbeiten offenbar in der Mehrzahl der Fälle zuverlässig: Für zurückliegende Trauerepisoden gilt in aller Regel, dass zwar rückblickend die Erinnerung daran bleibt, wie sehr man getrauert hat, diese Trauer aber aktuell nicht mehr schmerzt. Offenbar hat sich, gewissermaßen hinter der Bühne des Bewusstseins, die interne Organisation von Wünschen und Bewertungen verändert, entwickelt, angepasst. Hinzu kommt, dass der akkommodative Modus erkennbar kein einzelner Prozess ist, sondern eine breite Kategorie unterschiedlicher Prozesse umfasst (Brandtstädter 2007; Thomsen 2016). Oft wird eine Kombination von Prozessen zusammengenommen dazu führen, dass der Person das blockierte Ziel nicht mehr so wichtig erscheint wie früher. Dazu gehört typischerweise das Loslassen des alten Ziels, vielfach auch das Umorientieren, vielleicht aber auch nur die Änderung der Perspektive auf das bisherige Ziel (etwa indem der Maßstab für „erfolgreich" auf andere Vergleichsgruppen bezogen wird) oder die Aufwertung einer Alternative, die nun attraktiver erscheint. Manchmal genügt es, die Perspektive zu weiten („Das, was mir widerfahren ist, ist bedrückend und schmerzlich - aber vielen anderen Menschen ist viel Schlimmeres widerfahren!"). Dabei ist noch ungeklärt, wie sich die „Auswahl“" zwischen diesen verschiedenen Prozessen abspielt (Greve und Thomsen 2019b). Festhalten lässt sich indessen allemal, dass beispielsweise Prozesse der Veränderung von Vergleichsmaßstäben bei Strafgefangenen eine Rolle zu spielen scheinen (mit Auswirkungen auf die Anwendbarkeit testpsychologischer Inventare: Steller 1983).

Eine Reihe von Befunden (Überblick: Brandtstädter und Rothermund 2002; Heckhausen et al. 2010, Haase et al. 2013) zeigt, dass es erhebliche interindividuelle Unterschiede in der Bereitschaft oder Fähigkeit gibt, akkommodative Anpassungsreaktionen zu zeigen. Dies verweist darauf, dass 
die individuelle „Akkommodativität“ von Entwicklungsverläufen und -bedingungen abhängt (Meyer und Greve 2012). Eben hier setzen Überlegungen an, wie die individuelle Bereitschaft zur Zielanpassung gefördert werden könnte, um dadurch auch die Prozesse der Selbstregulation zu verbessern (Greve und Thomsen 2019b). Im Zweiprozessmodell der Entwicklungsregulation werden Annahmen zu drei Aspekten gemacht, die interindividuelle Unterschiede in der „Akkommodativität“ erklären können. Zunächst kann ein facettenreiches, heterogenes Selbstkonzept als Voraussetzung dafür angesehen werden, alternative Ziele überhaupt wahrzunehmen und attraktiv zu finden. Allerdings reicht das alleinige Generieren von alternativen Ziele nicht aus, denn schließlich muss das Individuum auch in der Lage sein, das alte Ziel durch ein neues zu ersetzen (Wrosch et al. 2003) und entlastende Kognitionen verfügbar haben, die dabei unterstützen, mit der getroffenen Entscheidung zufrieden zu sein (Brandtstädter und Rothermund 2002). Auf den hiesigen Kontext bezogen wurde schon erwähnt, dass in der Lesart der Theorien der kognitiven Transformation der Ausstieg aus einer intensiven kriminellen Karriere ja v.a. eine kohärente und individuell zufriedenstellende Transformation des Selbstkonzeptes von einer Identität als krimineller Mensch in ein neues Selbstbild impliziert (Bliesener und Lösel 2002). Erste Befunde (aus früheren Entwicklungsphasen) deuten darauf hin, dass beispielsweise heterogene Erfahrungskontexte die notwendige kognitive Flexibilität erhöhen könnten (Greve und Thomsen 2013, Thomsen und Greve 2013). Ob auch belastende oder kritische Lebensereignisse die Entwicklung akkommodativer Prozesse vorantreiben können, z. B. im Sinne posttraumatischen Wachstums (Calhoun und Tedeschi 2006), ist bislang kaum untersucht worden (Meyer und Greve 2012). Wenn die adaptive Dynamik von Ziel- und Intentionswahlen eine wichtige Voraussetzung für besser gelingende Selbst- und Entwicklungsregulation im Erwachsenenalter sein sollte, läge hier ein wichtiger, bislang kaum genutzter Ansatzpunkt für gezielte Interventionsansätze im Strafvollzug, der die Perspektive der (aktionalen) Selbstgestaltung und der adaptiven Zielwahl dafür systematisch ernst nimmt.

Hierfür ist jedoch auch die Beachtung solcher Regulationsprozesse erforderlich, die nicht nur die Umsetzung von Zielen, sondern auch ihre Bewertung und Veränderung beeinflussen; dies umfasst neben den angesprochenen volitionalen Dynamiken insbesondere auch emotionale Prozesse.

\section{Emotionen und ihre verhaltenssteuernde Funktion}

Emotionsregulative Prozesse beeinflussen sowohl die Zielverfolgung als auch die Zielanpassung und offerieren auch einen möglichen Zugang zur Erklärung der Steuerung akkommodativer Prozesse. Auch wenn dies theoretisch bislang noch nicht weiter ausgeführt wurde, bietet, wie im
Folgenden ausgeführt wird, insbesondere die Persönlichkeits-System-Interaktionen(PSI)-Theorie von Kuhl (2001), einen Strukturrahmen, der es erlaubt, Prozesse der Zielbindung und Emotionsregulation miteinander zu verzahnen.

Erhebliche Anteile unseres Alltags verbringen wir demnach im Modus einer mehr oder weniger automatisierten Verhaltenssteuerung (,Autopilot“). Zur Aufgabenerfüllung greifen wir hierzu, sofern keine expliziten Handlungsintentionen vorliegen, auf etablierte Verhaltensroutinen zurück (Reizsteuerung) oder aber auf Verhaltensweisen, die andere von uns erwarten oder zu den wir uns gezwungen fühlen (Fremdsteuerung) (Kuhl 2001). Je nachdem, wie salient mögliche Intentionen, wie hoch die Motivation zur Zielverfolgung und wie eingefahren die Verhaltensroutinen sind, kann sich dies nicht nur darin bemerkbar machen, dass das man selbst mit dem Auto oder Fahrrad „plötzlich“ von Ort A nach B gefahren ist, ohne dass man die Einzelheiten der Fahrt vergegenwärtigen kann, sondern ggf. auch daran, dass z.B. ein Inhaftierter (auch ohne übermäßigen Affekt) schon wieder in eine körperliche Auseinandersetzung involviert wurde, ohne sich das Zustandekommen dieser Situation wirklich erklären zu können oder sie ,intendiert“ zu haben. Das Geschehen bleibt dann subjektiv unerklärlich, kann zwar kognitiv rekonstruiert und auch evaluativ eingeschätzt werden, wird aber nicht als Ergebnis aktiver Lebensgestaltung, bewusster Intention oder Zielverfolgung erlebt.

Setzt sich die betreffende Person in der Folge aber dann, intrinsisch motiviert, ein für sie vielleicht herausfordernde Entwicklungsziel (z. B. in den nächsten 6 Monaten nicht mehr in eine risikoreiche Auseinandersetzungen zu geraten, sondern im Konfliktfall auszuweichen oder zumindest nicht aktiv anzugreifen), wird dieses Ziel nur dann im Gedächtnis salient und damit bewusst verfolgbar bleiben, wenn die damit einhergehenden negativen Affekte bzw. Frustrationen (Verlust an Ansehen vor den Mitgefangenen; Angst, selbst zum Opfer zu werden; Angst, es nicht zu schaffen) zunächst einmal ausgehalten und nicht durch Suppression abgewehrt werden müssen. Dabei geht mit dem aktivierten negativen Affekt eine Wahrnehmungseinengung einher, die den Blick vom Gesamtzusammenhang (intendiertes Entwicklungsziel und Verhaltensumsetzung) auf singuläre Probleme und Einzelheiten lenkt, sodass die übergeordnete Änderungsmotivation verloren gehen kann und dysfunktionale Regulationsstrategien wie Rumination die Oberhand gewinnen können. Sobald die Intention im Gedächtnis fest repräsentiert ist, ist es daher erforderlich, möglichst rasch in eine positive affektive Stimmung zu kommen, welche die Willensanbahnung und Umsetzung der Intention in Handlungen begünstigt. Hingegen würde ein bleibender negativer Affekt die Handlungsumsetzung behindern. Die Fähigkeit, nach Bedarf und Erfordernis negative Affekte auszuhalten, sich aber möglichst schnell zu regenerieren und gezielt in 
eine positive Stimmung zu versetzen, die zur weiteren Motivation und Handlungsausführung beiträgt, ist daher ein wesentliches Element erfolgreicher Selbst- bzw. Emotionsregulation (Kuhl 2001).

Dies führt vor Augen, dass bei der Umsetzung von Absichten in Handlungen und dem Lernen aus Fehlern sowie der Verhaltensänderung Emotionen und emotionsregulative Prozesse eine zentrale Rolle spielen. Vor allem die Regulation negativer und positiver Affekte, d.h. auch das Ausmaß der Erregung und die Anspannung bzw. Regenerationsgeschwindigkeit, sind für erfolgreiche Selbstregulation und Selbstwachstum zentral. Hinzu kommt die Flexibilität der Aufmerksamkeitslenkung auf den Kontext bzw. das Selbst. Ein zu starker oder zu lange anhaltender negativer Affekt behindert die angemessene Berücksichtigung des Gesamtzusammenhangs. d.h. des eigenen biografischen Werdegangs und des eigenen Lebensnarrativs, was selbstbestimmtes Handeln und Ich-Integrität (im Sinne von Erikson 1966 [1959]) erschwert oder unmöglich macht. Damit ermöglicht ein Fokus auf die emotionsregulativen Prozesse nicht nur den Brückenschlag zu entwicklungspsychologischen Theorien, sondern auch zu den affektiven Neurowissenschaften, welche davon ausgehen, dass der ,emotionale Stil“" eines Menschen (Davidson und Begley 2012) Aussagen darüber erlaubt, wie häufig, intensiv und anhaltend positive und negative Affekte erlebt werden und wie stark das Individuum dadurch in der Verhaltenssteuerung behindert wird.

Beispielsweise sind im Hinblick auf den spezifischen Entwicklungskontext Strafvollzug die ,,moralischen“ Emotionen Scham und Schuld und deren Regulation von besonderer Bedeutung. Scham und Schuld bilden im Zusammenspiel mit Empathie und kognitiven moralischen Überzeugungen das Gerüst des menschlichen Gewissens und sind damit insbesondere, aber eben nicht ausschließlich, für das Entwicklungsziel der Resozialisierung relevant; sowohl, was die Zielentwicklung als auch die Zielverfolgung betrifft. Setzt sich beispielsweise ein Inhaftierter das Ziel, künftig straffrei zu bleiben, wird dies u.a. auch negative Affekte, wie z.B. Angst vor dem Scheitern oder Verunsicherung im Hinblick auf das bisherige Selbstbild, mit sich bringen, die reguliert werden müssen. Eine Emotionsregulation in eine motivationspsychologisch günstigere, positive Ausgangslage wird jedoch dadurch erschwert, dass im Verlauf der Haft und Behandlung u. a. Scham- und Schuldgefühle, d.h. zusätzliche negative Affekte, in besonderer Weise durch den Kontext induziert und salient werden (Ewald 2018; Hosser et al. 2005, 2008). Diese können sich direkt auf das eigene kriminelle Handeln oder das bisherige Scheitern an der Straffreiheit beziehen oder sie entstehen z.B. auch aus der Auseinandersetzung mit den Folgen des eigenen Verhaltens für nahestehende Personen, die von den Sanktionsfolgen mitbetroffen sind. Das Erleben von posi- tiver Affektivität, z.B. durch authentischen Stolz auf eine selbst erzielte Leistung, ist im Kontext des Gefängnisses und subkultureller Bestrebungen hingegen vermutlich eher selten, was die Zielumsetzung behindert, sofern nicht sehr gute emotionsregulative Kompetenzen vorhanden sind. So werden v. a. länger andauernde und/oder starke Schamgefühle in Zusammenhang mit Vermeidungsverhalten, sozialem Rückzug, Aggressivität, narzisstischem Verhalten und Delinquenz gebracht. Dabei wird vermutet, dass intensive Schamgefühle zu einem Selbstwertverlust führen und das Selbstkonzept durch eine verengte Betrachtungsperspektive eindimensionaler wird, was Abwehr- und Verteidigungsverhalten stimuliert sowie kompensierende Allmachtsfantasien, die ihrerseits zu unrealistischer Zielsetzung, mangelnder Motivation bei der Zielverfolgung, und Narzissmus führen können (Ciompi 2014). Infolgedessen steigt das Risiko negativer, ablehnender und sanktionierender Reaktionen des Umfeldes, welches wiederum zu erhöhter Aggressivität und häufigerem antisozialem Verhalten beitragen kann (Bliesener 1997). Emotionale und emotionsregulative Prozesse sind somit für die Zielbindung und Zielverfolgung zentral, wobei sie immer im Zusammenspiel mit dem aktuellen sozialen Kontext zu betrachten sind.

\section{Entwicklung in Kontexten: Entwicklungssysteme}

Auch wenn für bestimmte Lebensabschnitte charakteristische Entwicklungsaufgaben identifiziert werden können und es sicher aus praktischer Sicht oft sinnvoll ist, die Entwicklung einzelner Funktionsbereiche gesondert in den Blick zu nehmen (z.B. die Entwicklungsaufgaben im jungen Erwachsenenalter), sind die komplexe Vernetztheit und die wechselseitigen Abhängigkeiten dieser vielen verschiedenen Einzelaspekte auf verschiedenen Einflussebenen offensichtlich ein bestimmendes Merkmal von Entwicklung. Menschen sind komplexe, hierarchisch vielfach verschachtelte Systeme von interagierenden (Sub-)Systemen, die in ihrerseits verschachtelte Kontexte eingebettet sind. Eben diese Überlegung liegt dem Ansatz der ,Entwicklungssysteme“ zugrunde (z.B. Ford und Lerner 1992; Thelen und Smith 1998), der eben diese ,systemische“ Interdependenz von Entwicklungsprozessen betont (zusammenfassend Greve und Thomsen 2019a; in Bezug auf deviante Lebensläufe: Day und Wiesner 2019).

Im Anschluss an die klassischen Überlegungen von Bronfenbrenner (1981, 2005; zu einer erweiterten Version: Lerner 2002) umfasst diese systemische Perspektive den unmittelbaren (aktuelle soziale Bedingungen im Strafvollzug), den sozialen (z.B. Normen und Beziehungen in der Familie) sowie den allgemeinen Entwicklungskontext (gesellschaftliche Randbedingungen, z. B. Arbeitsmarktsituation, soziale Akzeptanz von Minderheiten etc.). Aus Sicht des je handelnden Individuums sind diese kontextuel- 
len Bedingungen für die Ziel- und Mittelauswahl zunächst als wahrgenommener Kontext bedeutsam (z. B.: Mittel, die mir aus meiner Sicht nicht gewährt werden, obwohl ich sie als erforderlich für eigene oder vorgegebene Ziele wahrnehme, müssen durch andere, ggf. auch unerlaubte ersetzt werden). Jedoch werden diese subjektiven Wahrnehmungen ihrerseits wesentlich auch durch Kontextbedingungen beeinflusst (z. B. begrenzt oder auch angeregt). Insbesondere kann der soziale Kontext (durch Personen ebenso wie Normen) Entwicklungsverläufe systematisch beeinflussen; neben den bereits erwähnten Auftretenswahrscheinlichkeiten für kritische Lebensereignisse betrifft dies auch für die Zielerreichung erforderlichen Mittel (Ressourcen Kompetenzen) sowie u.U. auch die Veränderung der (Prozesse der) Selbstregulation (etwa durch Maßnahmen der Straftäterbehandlung; Hosser und Boxberg 2014).

\section{Die Vollzugsanstalt als Entwicklungskontext}

Eine entwicklungspsychologische Perspektive auf den Strafvollzug wird also die Institution selbst als Lebens- und Entwicklungsraum ihrer Klientel in den Blick nehmen. Die Entwicklungspsychologie differenziert dabei üblicherweise unterschiedliche Kontexte, in denen sich Entwicklungsprozesse entfalten: beispielsweise den partnerschaftlichfamiliären Kontext, den Arbeitskontext, das Wohnumfeld oder auch den weiteren sozialen Kontext in der Freizeit und im Freundeskreis. Diese Kontexte sind gewöhnlich zumindest partiell voneinander abgegrenzt und bieten insoweit Möglichkeiten der gegenseitigen Kompensation, Unterstützung und Entlastung, beispielsweise in der Familie oder im Freundeskreis, wenn es im Arbeitskontext Probleme geben sollte. In diesem Sinne können abgegrenzte Kontexte also auch eine Entwicklungsressource sein. Darüber hinaus sind die Kontexte - jedenfalls in Maßen - selbst gewählt und partiell gestaltbar und insoweit zumindest teilweise an eigene Wünsche, Bedürfnisse und Ziele anpassbar.

Hier stellt eine Vollzugsanstalt - v.a. im geschlossenen Vollzug des sog. Regelvollzugs - ohne Zweifel einen hochspezifischen Entwicklungsrahmen dar, reguliert sie doch als „totale Institution“ (Goffman 1973) das Leben ihrer Insassen bis in Details und limitiert Reaktions- und Handlungsmöglichkeiten ebenso, wie sie verschiedene Handlungsund Anpassungsnotwendigkeiten bedingt (Hosser 2008). Sie definiert insoweit in einem einheitlichen Lebensraum mit einem einheitlichen Regelsystem eine Vielzahl derjenigen Lebenskontexte, die ansonsten abgrenzbare Lebenswelten darstellen, in denen Menschen sich normalerweise bewegen, agieren und entwickeln. So stehen der private Lebensraum (der Haftraum auf der Station mit eingeschränkten Rückzugsmöglichkeiten und Intimitätsräumen), die Lebensorganisation (mit vorgegebener Tagesstruktur, fremdbestimmter „Vollversorgung“ mit Nahrung, Wäschereinigung nach Wochenplan, regulierten Einkaufsmöglichkeiten und ärztlicher Versorgung durch einen Anstaltsarzt ohne Wahloption usw.), der engere soziale Kontext (die nicht selbst gewählten und gleichgeschlechtlichen Mitgefangenen und die ebenfalls nichtausgewählten Bediensteten auf der Station), der Arbeitskontext (mit den Mitgefangenen im Anstaltsbetrieb), ein Großteil des Freizeitbereichs (durch fremdorganisierte gemeinsame Freizeitangebote) unter der Kontrolle einer primär nach Sicherheitsprämissen organisierten Behörde, die unter denselben Gesichtspunkten auch die Kontakte mit Personen außerhalb der Anstalt und die individuellen Entscheidungsspielräume limitiert und überwacht.

Über dieses Regulatorium des Vollzugssystems hinaus ist der Insasse mit einem subkulturellen Regelsystem konfrontiert, das u. a. die Hierarchie in der Insassenschaft konstituiert und aufrechterhält, den Kontakt zum Anstaltspersonal limitiert oder Zugänge zu eingeschränkt verfügbaren oder illegalen Gütern kontrolliert (Bliesener und Klatt 2019; Klatt und Bliesener 2018). Diese beiden Normensysteme sind i. Allg. nicht kompatibel, sodass sich der Insasse in einem Normenkonflikt befindet. Die Anpassungsleistung in diesem Normenkonflikt gestaltet sich in der Regel so, dass der Gefangene versuchen wird, die erwarteten negativen Konsequenzen eines Normverstoßes in beide Richtungen zu minimieren. Dabei muss er die unterschiedlichen Sanktionierungsstrategien der beiden Regulationssysteme beachten. Während beide Systeme im Falle normkonformen Verhaltens mit - im lerntheoretischen Sinn - positiver Verstärkung (Vergabe von Vergünstigungen, Privilegien) arbeiten, kann das vollzugliche System v. a. mit negativer Verstärkung (Lockerungen, vorzeitige Entlassung) und negativer Bestrafung (Entzug von Vergünstigungen, Lockerungen) reagieren. Positive Bestrafungen (Übelzufügungen) stehen dem vollzuglichen Regulatorium nur eingeschränkt zur Verfügung, sind aber im subkulturellen System, beispielsweise in Form von Gewalt, gängiges Mittel der verhaltenssteuernden Einflussnahme (Bliesener 2014c; Bliesener und Thomas 2012; Hosser und Taefi 2008). Es wurde bereits darauf hingewiesen, dass hierdurch Anpassungsnotwendigkeiten erwachsen, die potenziell geeignet erscheinen, den Gefangenen von der Beschäftigung mit eigentlich anstehenden Problemen und Entwicklungsaufgaben abzuhalten, die hierdurch angestoßenen Entwicklungen für die Zeit nach der Haft aber möglicherweise nur wenig nützlich sind.

Von einem übergeordneten Standpunkt aus gesehen, ist der Motor für Entwicklungsprozesse die Auseinandersetzung mit Problemen, unabhängig davon, ob man die Probleme nun als normative Entwicklungsaufgabe, als nonnormatives Anpassungserfordernis an veränderte Rahmenbedingungen oder als wahrgenommene Ist-Ziel-Diskrepanz 
konzipiert. Für ihre Lösung werden Ressourcen benötigt, die internal (Kompetenzen) oder external (Unterstützung, Bereitstellung von Möglichkeiten) sein können. Auf einige gehäuft bei einer straffälligen und inhaftierten Zielgruppe zu erwartenden Besonderheiten internaler Ressourcen wurde bereits verschiedentlich eingegangen. Auch auf Besonderheiten der Zurverfügungstellung externer Ressourcen im Kontext einer Strafvollzugsanstalt wurde bereits eingegangen. Dabei wurde bislang aber einseitig ihre Limitierung betont. Tatsächlich aber bietet der Kontext einer Vollzugseinrichtung auch Ressourcen, die zumindest einigen Gefangenen ansonsten wahrscheinlich nicht zur Verfügung gestanden hätten. Neben der an vollzuglichen und Sicherheitserfordernissen oder an punitiven Gesichtspunkten orientierten restriktiven Facette, bietet die dem Resozialisierungsauftrag verpflichtete Institution ja auch Hilfeangebote; sei es in Form therapeutischer Maßnahmen, schulischer oder beruflicher Qualifikationsangebote oder der Unterstützung bei der vorbereitenden Organisation eines strukturierten Entlassungsumfeldes (Hosser und Boxberg 2014). Viele dieser Angebote bezwecken die Verbesserung der internen Ressourcen des Gefangenen, andere die Linderung der Probleme beim Weg aus der Haftanstalt zurück in die Gesellschaft (Hosser et al. 2007). Ihre Sichtung und Organisation (auch) unter entwicklungspsychologischen Gesichtspunkten könnte lohnenswert sein.

Es lassen sich aber auch informelle externe Ressourcen ausmachen. So ist eine Vollzugsanstalt ja nicht nur ein Sicherheitsapparat, sondern es arbeiten dort Menschen, die mitunter Beziehungsangebote machen, im Alltag und bei großen Themen Unterstützung bieten, Vorbilder sein können. Dies gilt auch für die Mitgefangenen, die ja nicht nur Mitglieder einer Subkultur im Vollzugsalltag sind, sondern auch soziale Wesen mit Bedürfnissen, aber auch Kompetenzen und Ressourcen, die sie mitunter zu teilen bereit sind (Hosser 2001). Dieser Kosmos definiert insoweit einen Möglichkeitsraum mit Begrenzungen und teilweise sehr speziellen Chancen, in dem Veränderungs- und Entwicklungsprozesse stattfinden sollen.

\section{Schlussfolgerung: die Vollzugsanstalt als Entwicklungsraum}

Die Vollzugsanstalt wurde einleitend als Entwicklungsintervention definiert, die auf den Gefangenen gezielt einwirken soll, um dessen Resozialisierungschancen im Sinne eines zukünftig straffreien Lebens zu erhöhen. Es wurde aufgezeigt, dass dieser Resozialisierungsauftrag historisch bislang weitgehend zunächst als Erziehungsauftrag, dann ergänzend und zunehmend auch als Behandlungsauftrag interpretiert wurde. Dabei wurde argumentiert, dass diese Art der Umsetzung anhand von Methoden und Konzep- ten, die Veränderung von vornherein mit Blick auf ein von außen gesetztes Ziel betrachten, Grenzen aufweist, da sie relevante Anteile ihrer Zielgruppe nicht erreicht, mitunter auch kontraintentionale Wirkungen erzielt. Es wurde daher eine Erweiterung der Perspektive auf die Vollzugsanstalt als Entwicklungsintervention vorgeschlagen, die den Gefangenen weniger - zumindest nicht ausschließlich - aus dem Blickwinkel eines gewünschten Endzustands betrachtet und nach der „,best practice“ fragt, wie dieser am effizientesten zu erreichen sein mag (,what works"). Vielmehr ist zunächst grundsätzlicher nach den Voraussetzungen, Möglichkeiten und Hemmnissen für Veränderungsprozesse bei der speziellen Zielgruppe strafgefangener Menschen in dem speziellen Kontext einer Strafvollzugsanstalt zu fragen. Hierzu wurde vorgeschlagen, sich entwicklungspsychologischer Konzepte, Erkenntnisse und Modelle zu bedienen, weil die Entwicklungspsychologie derjenige Wissenschaftszweig ist, der menschliche Veränderungsprozesse über die Lebensspanne zum Gegenstand hat und nicht notwendig von vornherein einen vordefinierten Zielzustand im Blick hat. Es wurde sodann der Versuch unternommen, ein entwicklungspsychologisches Rahmengerüst für die Untersuchung des Strafvollzugs und ihrer Klientel zu entwerfen. Hierfür wurden zentrale und potenziell geeignete entwicklungspsychologische Konzepte auf ihre Geeignetheit für diesen Zweck und auf ihre Anschlussfähigkeit zu kriminalpsychologischen Konzepten hin untersucht. Insoweit sich Veränderung aus dem Blickwinkel der Entwicklungspsychologie entlang der Auseinandersetzung mit Problemen vollzieht, die sich einem Menschen im Laufe seines Lebens stellen, wurden diese aus der Perspektive (1.) normativer, sich also in bestimmten Lebensphasen typischerweise stellender „Entwicklungsaufgaben“, (2.) nonnormativer, sich aufgrund ,kritischer Lebensereignisse“ stellender Anpassungserfordernisse, und (3.) selbst gewählter relevanter Lebensziele, auf deren Diskrepanz mit dem wahrgenommenen Istzustand zu reagieren ist, untersucht. Dabei zeigte sich, dass die spezielle Klientel im Justizvollzug in der speziellen Entwicklungsumgebung „Gefängnis“ im Vergleich zur Durchschnittsbevölkerung Besonderheiten erwarten lässt, deren systematische Beforschung aus Sicht der Autoren lohnenswert sein könnte. Weiterhin zeigte es sich, dass viele der aus diesen Analysen erwachsenen Hypothesen gut mit kriminologischen und kriminalpsychologischen Theorien und Befunden kompatibel sind.

Nun hat die Justizvollzugsanstalt auf der anderen Seite einen klaren gesellschaftlichen Resozialisierungsauftrag, der insoweit ja tatsächlich von einem gewünschten Zielzustand ausgeht und der impliziert, dass die Gefangenen, da sie diesen Zustand aufgrund der Tatsache ihrer Inhaftierung offenbar bislang noch nicht erreicht haben, hier Defizite aufweisen, die im Sinne. einer ,correctional institution“ zu korrigieren sind. Der mit dem hiesigen Vorschlag zur Per- 
spektiverweiterung einhergehende Apell, zunächst ergebnisoffener die Inhalte, Voraussetzungen, Hindernisse und Chancen für Veränderungsprozesse strafgefangener Menschen in den Blick zu nehmen, heißt jedoch nicht, dass es unmöglich wäre, etwaige Erträgnisse aus einer solchen (Forschungs-)Perspektive für Überlegungen über Möglichkeiten gezielter Entwicklungsinterventionen zu nutzen. Hier wird man zunächst sicherlich die Vollzugseinrichtung selbst in den Blick nehmen und, jenseits ihres Sicherungsauftrags, nach Möglichkeiten fahnden, entwicklungspsychologisch fundiert Entfaltungsmöglichkeiten auszuloten, um die Einrichtung, wo immer dies möglich ist, zu einem Raum zu gestalten, der Entwicklungsprozesse ihrer Klientel anregt, fördert und ermöglicht. Aber auch personenbezogene Interventionsansätze sind denkbar. Warum sollte man beispielsweise nicht darüber nachdenken, ob man einer hochgradig psychopathologisch und kriminologisch vorbelasteten Kerngruppe Sicherungsverwahrter mit ausgeprägter Hafterfahrung und vielfältig gescheiterten Behandlungsversuchen vielleicht nicht ein entwicklungspsychologisch fundiertes "Coaching“ anbietet, nach realistischen Lebenszielen und dem Weg dorthin zu suchen, anstatt nach dem Prinzip des immer „Mehr-vom-Selben“ stets aufs Neue Behandlungsangebote zu unterbreiten, die er bereits vielfältig versucht und an denen er bislang stets gescheitert ist? Die Aufgabe, die sich diesen Menschen aus einer entwicklungspsychologischen Perspektive im Hinblick auf die Zielverfolgung und Transformation ihres Selbstbildes stellt, wurde bereits angesprochen. Es besteht insoweit die begründete Hoffnung, durch eine Perspektivenausweitung vielleicht die Grenzen des bisherigen risikofokussierten und wenig an Erklärung orientierten Resozialisierungsmodells (Ward 2020) ein Stück ausweiten zu können.

Auch wenn die Konnotation der einleitend zitierten Typologie von Liszts (,,besserungsfähig und -willig“, „einschüchterungsfähig“, ,unverbesserlich“) sicher weder angemessen noch fruchtbar ist, ist es vielleicht doch lehrreich, nach anschlussfähigen Momenten seines Gedankens zu suchen. Eine Abschreckung (derer, die durch sie erreicht werden) nimmt ja eigentlich eine aktualgenetische, motivationale Perspektive auf straffälliges Verhalten ein: Ihre Rationale ist es, dem Täter andere, bessere Gründe zu geben, kriminelles Handeln (d.h.: intentionales Verhalten: Suhling und Greve 2010) künftig zu unterlassen. Die Erziehung und Behandlung (derer, die sich mit den uns bekannten Mitteln zum aus unserer Sicht Besseren verändern lassen - können und wollen) nehmen dagegen die ontogenetische Perspektive ein: Ihr Ziel ist es, den Tätern die Fähigkeiten zu geben, kriminelles Handeln zukünftig zu unterlassen. Das kann etwa die Regulation von Emotionen implizieren (die es erschweren oder verhindern, dass man den eigentlich eingesehenen Gründen auch tatsächlich folgen kann), oder es kann die Fähigkeit betreffen, die zu den eigenen
Möglichkeiten besser passenden Ziele tatsächlich für erstrebenswert zu halten. Die Einordnung als „unverbesserlich“ ist hingegen immer relativ zu den je verfügbaren Möglichkeiten, so wie früher unheilbare Erkrankungen es zu einem späteren Zeitpunkt nicht mehr sind. Diese hängen wiederum v. a. davon ab, ob wir zutreffende Erklärungen für die aktuelle individuelle Konstellation (insbesondere von regulativen Kompetenzen) haben. Eben hierfür, nach diesen Erklärungen aus entwicklungspsychologischer Perspektive zu suchen, haben wir geworben. Der Entwicklungsraum, den die Strafhaft trotz und wegen der mit ihr verbundenen Restriktionen und Sicherungsaufgaben darstellt und bietet, kann dafür aus Sicht der Autoren genutzt werden, wenn die dynamische Perspektive systematischer verfolgt wird.

Funding Open Access funding provided by Projekt DEAL.

Interessenkonflikt K.-P. Dahle, W. Greve, D. Hosser und T. Bliesener geben an, dass kein Interessenkonflikt besteht.

Open Access Dieser Artikel wird unter der Creative Commons Namensnennung 4.0 International Lizenz veröffentlicht, welche die Nutzung, Vervielfältigung, Bearbeitung, Verbreitung und Wiedergabe in jeglichem Medium und Format erlaubt, sofern Sie den/die ursprünglichen Autor(en) und die Quelle ordnungsgemäß nennen, einen Link zur Creative Commons Lizenz beifügen und angeben, ob Änderungen vorgenommen wurden.

Die in diesem Artikel enthaltenen Bilder und sonstiges Drittmaterial unterliegen ebenfalls der genannten Creative Commons Lizenz, sofern sich aus der Abbildungslegende nichts anderes ergibt. Sofern das betreffende Material nicht unter der genannten Creative Commons Lizenz steht und die betreffende Handlung nicht nach gesetzlichen Vorschriften erlaubt ist, ist für die oben aufgeführten Weiterverwendungen des Materials die Einwilligung des jeweiligen Rechteinhabers einzuholen.

Weitere Details zur Lizenz entnehmen Sie bitte der Lizenzinformation auf http://creativecommons.org/licenses/by/4.0/deed.de.

\section{Literatur}

Ahnert A (Hrsg) (2014) Theorien der Entwicklungspsychologie. Springer VS, Berlin

Baltes PB (1987) Theoretical propositions of life-span developmental psychology: On the dynamics between growth and decline. Dev Psychol 23:611-626

Baltes PB, Baltes MM (1990) Psychological perspectives on successful aging: the model of selective optimization with compensation. In: Baltes PB, Baltes MM (Hrsg) Successful aging. Perspectives from the behavioral sciences. Cambridge University Press, Cambridge, S 1-34

Baltes PB, Lindenberger U, Staudinger UM (2006) Life-span theory in developmental psychology. In: Lerner RM (Hrsg) Theoretical models of human development, 6. Aufl. Handbook of child psychology, Bd. 1. Wiley, New York, S 569-664

Barth TL (2015) Partnerschaft und Sexualität inhaftierter Männer im deutschen Strafvollzug. Unveröffentlichte Dissertation, Charité Universitätsmedizin Berlin

Bliesener T (1997) Konflikte und Aggressionen zwischen Jugendlichen: Zusammenhänge mit situationsbezogenen Informationsverarbeitungen und Reaktionsmustern. In: Bundeskriminalamt (Hrsg) Informationen aus der kriminalistisch-kriminologischen Forschung - Forum 1996. Bundeskriminalamt, Wiesbaden, S 97-125 
Bliesener T (2003) Jugenddelinquenz. Risikofaktoren, Prävention, Intervention und Prognose. Prax Rechtspsychol Sonderh Jugenddelinq 13:174-191

Bliesener T (2009) Junge Mehrfach- und Intensivtäter - Definitionen, Hintergründe und Konzepte der Prävention. Z Soz Strafrechtspfl $18: 13-24$

Bliesener T (2010) Der Umgang mit jungen Mehrfach- und Intensivtätern - Probleme der Definition, Prävention und Intervention. Bewährungshilfe 57:357-371

Bliesener T (2012) Persistent juvenile offenders. In: Bliesener T, Beelmann A, Stemmler M (Hrsg) Antisocial behavior and crime: contributions of developmental and evaluation research to prevention and intervention. Hogrefe, Göttingen, S 53-68

Bliesener T (2014a) Jugenddelinquenz. In: Bliesener T, Lösel F, Köhnken G (Hrsg) Lehrbuch Rechtspsychologie. Huber, Bern, S 64-75

Bliesener T (2014b) Erklärungsmodelle dissozialen Verhaltens. In: Bliesener T, Lösel F, Köhnken G (Hrsg) Lehrbuch Rechtspsychologie. Huber, Bern, S 37-63

Bliesener T (2014c) Sanktionen und Sanktionswirkung. In: Melzer W, Hermann D, Sandfuchs U, Schäfer M, Schuberth W, Daschner P (Hrsg) Handbuch Aggression, Gewalt und Kriminalität bei Kindern und Jugendlichen. Klinkhardt, Bad Heilbrunn, S 92-96

Bliesener T (2017) Resilienz - Schutzfaktoren für delinquentes Handeln. In: Hermann D, Pöge A (Hrsg) Handbuch der Kriminalsoziologie. Nomos, Baden-Baden, S 267-282

Bliesener T, Klatt T (2019) Der Jugendarrest in Niedersachsen und Schleswig-Holstein nach Inkrafttreten der Landesvollzugsgesetze. Unsere Jugend 71:465-472

Bliesener T, Lösel F (2002) Identitätsbildung, Gruppenstruktur und Gruppenerleben bei Hooligans. In: Herzog M (Hrsg) Fußball als Kulturphänomen. Kunst - Kultur - Kommerz. Kohlhammer, Stuttgart, S 253-268

Bliesener T, Thomas J (2012) Wirkt Strafe, wenn sie der Tat auf dem Fuße folgt? Zur psychologisch-kriminologischen Evidenz des Beschleunigungsgebots. Z Jugendkriminalr Jugendh 23:382-389

Boerner B, Jopp D (2007) Improvement/maintenance as reorientation as central features of coping with major life change and loss: contributions of three life-span theories. Hum Dev 50:171-195

Bonta J, Andrews D (2017) The psychology of criminal conduct, 6. Aufl. Routledge, New York

Brandtstädter J (1990) Entwicklung im Lebensablauf. Ansätze und Probleme der Lebensspannen-Entwicklungspsychologie. Kölner Z Soziol Sozialpsychol 31:322-350

Brandtstädter J (2001) Entwicklung - Intention - Handeln. Kohlhammer, Stuttgart

Brandtstädter J (2006) Action perspectives on human development. In: Lerner RM (Hrsg) Theoretical models of human development, 6. Aufl. Handbook of child psychology, Bd. 1. Wiley, New York, S $516-568$

Brandtstädter J (2007) Das flexible Selbst: Selbstentwicklung zwischen Zielbindung und Ablösung. Elsevier, München

Brandtstädter J (2015) Positive Entwicklung. Zur Psychologie gelingender Lebensführung, 2. Aufl. Springer, Berlin

Brandtstädter J, Greve W (2006) Entwicklung und Handeln: Aktive Selbstentwicklung und Entwicklung des Handelns. In: Schneider W, Wilkening F (Hrsg) Entwicklungspsychologie: Theorien. Enzyklopädie der Psychologie, Bd. C/V/1. Hogrefe, Göttingen, S 409-459

Brandtstädter J, Lerner RM (Hrsg) (1999) Action and self-development: theory and research through the life span. SAGE, Thousand Oaks

Brandtstädter J, Lindenberger U (Hrsg) (2007) Entwicklungspsychologie der Lebensspanne. Kohlhammer, Stuttgart

Brandtstädter J, Rothermund K (2002) The life-course dynamics of goal pursuit and goal adjustment: a two-process framework. Dev Rev 22:117-150. https://doi.org/10.1006/drev.2001.0539

Bronfenbrenner U (1981) Die Ökologie der menschlichen Entwicklung: natürliche und geplante Experimente. Klett-Cotta, Stuttgart
Bronfenbrenner U (2005) Making human beings human. SAGE, Thousand Oaks

Calhoun LG, Tedeschi RG (Hrsg) (2006) Handbook of posttraumatic growth. Erlbaum, Mahwah, NJ

Chaiken S, Trope Y (Hrsg) (1999) Dual-process theories in social psychology. Guilford, New York

Ciompi L (2014) Die Bedeutung von verletzten Selbstwertgefühlen und Scham in der Sozialpsychiatrie. Lead Opin Latros Neurol Psychiatr 1:40-42

Dahle K-P (1998) Straffälligkeit im Lebenslängsschnitt. In: Kröber H-L, Dahle K-P (Hrsg) Sexualstraftaten und Gewaltdelinquenz. Kriminalistik Verlag, Heidelberg, S 47-54

Dahle K-P (2001) Violent crime and offending trajectories in the course of life: an empirical life span developmental typology of criminal careers. In: Farrington DP, Hollin C, McMurran M (Hrsg) Sex and violence: the psychology of crime and risk assessment. Routledge, London, New York, S 197-209

Dahle K-P (2005a) Delinquenzverläufe über die Lebensspanne: Anwendungsperspektiven einer entwicklungsorientierten Sichtweise. In: Dahle K-P, Volbert R (Hrsg) Entwicklungspsychologische Aspekte der Rechtspsychologie. Hogrefe, Göttingen, S 79-91

Dahle K-P (2005b) Psychologische Kriminalprognose - Wege zu einer integrativen Prognosemethode. Centaurus, Herbolzheim

Dahle K-P (2010) Die Begutachtung der Gefährlichkeits- und Kriminalprognose des Rechtsbrechers. In Volbert R, Dahle K-P (Hrsg) Forensisch-psychologische Diagnostik im Strafverfahren. Göttingen, Hogrefe, S 67-114

Dahle K-P (2019) Die Maßregel der Sicherungsverwahrung nach den Urteilssprüchen des EGMR und BVerfG - Challenge oder eher Mission Impossible? Vortrag auf dem Bundesweitem Forum Sicherungsverwahrung, Celle, 4.-5.11.2019

Dahle K-P, Lehmann RBJ (2018) Zum prognostischen Mehrwert einer integrativen nomothetisch-idiografischen kriminalpsychologischen Prognosebeurteilung - Eine empirische Untersuchung an männlichen Gewalt- und Sexualstraftätern. Forens Psychiatr Psychol Kriminol 12:37-50

Dahle K-P, Volbert R (Hrsg) (2005) Entwicklungspsychologische Aspekte der Rechtspsychologie. Hogrefe, Göttingen

Dahle K-P, Lohner JC, Konrad N (2005a) Suicide prevention in penal institutions: validation and optimization of a screening tool for early identification of high-risk inmates in pretrial detention. Int $\mathbf{J}$ Forensic Ment Health 4:53-62

Davidson RJ, Begley S (2012) The emotional life of your brain. Hudson Street Press, New York

Day DM, Wiesner M (2019) Criminal trajectories. A developmental perspective. University press, New York

Endres J, Breuer MM (2018) Behandlungsmaßnahmen und -programme im Strafvollzug. In: Maelicke B, Suhling S (Hrsg) Das Gefängnis auf dem Prüfstand. Zustand und Zukunft des Strafvollzugs. Springer, Berlin, S 89-108

Erikson EH (1966) Identität und Lebenszyklus. Suhrkamp, Frankfurt a.M.

Erikson EH (1982) The life cycle completed: a review, 1. Aufl. Norton, New York

Ewald E (2018) Selbstbewertende Emotionen und Delinquenz-Scham, Schuld, Peinlichkeit und Stolz bei Straftätern. Dissertation. TU Braunschweig, Braunschweig (https://publikationsserver. tu-braunschweig.de/receive/dbbs_mods_00066116)

Farrington DP, Kazemian L, Piquero AR (Hrsg) (2019) The Oxford handbook of developmental and life-course criminologiy. Oxford University Press, Oxford

Featherman DL, Lerner RM (1985) Ontogenesis and sociogenesis: problematics for theory and research about development and socialization across the life span. Am Sociol Rev 50:659-676

Filipp S-H, Aymanns P (2010) Kritische Lebensereignisse - eine Annäherung. In: Filipp S-H, Aymanns P (Hrsg) Kritische Lebensereignisse und Lebenskrise. Kohlhammer, Stuttgart 
Ford DH, Lerner RM (1992) Developmental systems theory: an integrative approach. SAGE, Thousand Oaks

Freund A (2008) Successful aging as a management of ressources: the role of selection, optimization, and compensation. Res Hum Dev 5:94-106

Freund AM, Baltes PB (2005) Entwicklungsaufgaben als Organisationsstrukturen von Entwicklung und Entwicklungsoptimierung. In: Filipp SH, Staudinger UM (Hrsg) Enzyclopädie der Psychologie: Entwicklungspsychologie, Bd 6. Hogrefe, Göttingen, S $35-78$

Freund AM, Li KZH, Baltes PB (1999) Successful development and aging. The role of selection, optimization, and compensation. In: Brandtstädter J, Lerner RM (Hrsg) Theory and research through the life span. SAGE, Thousand Oaks, S 401-434

Gautschie HR (1956) Die Erziehung im Strafvollzug. Z Präventivmed 1:467-478

Giordano P, Cernkovich S, Rudolph J (2002) Gender, crime, and desistance: toward a theory of cognitive transformation. Am J Sociol 107:990-1064

Goffman E (1973) Asyle - Über die soziale Situation psychiatrischer Patienten und anderer Insassen. Suhrkamp, Frankfurt, a.M.

Greve W (2007) Selbst und Identität im Lebenslauf. In: Brandtstädter J, Lindenberger U (Hrsg) Entwicklungspsychologie der Lebensspanne. Kohlhammer, Stuttgart, S 305-336

Greve W, Enzmann D (2003) Self-esteem maintenance among incarcerated young males. Int J Behav Dev 27:12-20

Greve W, Leipold B (2018) Intentionale Selbstgestaltung und Problembewältigung. In: Schneider W, Lindenberger U (Hrsg) Entwicklungspsychologie, 8. Aufl. Beltz, Weinheim, S 579-594

Greve W, Thomsen T (2013) Developmental conditions of adaptive self-stabilization in adolescence: an exploratory study. Int J Dev Sci 7:119-131

Greve W, Thomsen T (2019a) Entwicklungspsychologie. Eine Einführung in die Erklärung menschlicher Entwicklung. Springer, Berlin

Greve W, Thomsen T (2019b) Die Entwicklung der Selbstregulation über die Lebensspanne. In: Deing P, Riemann S (Hrsg) Psychologie der Selbststeuerung. Springer, Berlin, S 3-21

Greve W, Enzmann D, Hosser D (2001) The stabilization of selfesteem among incarcerated adolescents: processes of accommodation and immunization. Int $\mathbf{J}$ Offender Ther Comp Criminol 45:749-768

Grieger L, Hosser D, Schmidt AF (2012) Predictive validity of self-reported self-control for different forms of recidivism. J Crim Psychol 2:80-95

Haase CM, Heckhausen J, Wrosch C (2013) Developmental regulation across the life span: toward a new synthesis. Dev Psychol 49(5):964-972

Havighurst RJ (1972) Developmental tasks and education. Longman, New York

Heckhausen J, Wrosch C, Schulz R (2010) A motivational theory of life-span development. Psychol Rev 117:32-60

Heckhausen J, Wrosch C, Schulz R (2019) Agency and monitvation in adulthood and old age. Annu Rev Psychol 70:191-217

Hosser D (2001) Soziale Unterstützung im Strafvollzug. Hafterleben und protektive Faktoren bei jungen Männern. Nomos, Baden-Baden

Hosser D (2008) Prisonisierungseffekte. In: Steller M, Volbert R (Hrsg) Handbuch der Rechtspsychologie. Hogrefe, Göttingen, S $172-179$

Hosser D, Boxberg V (2014) Intramurale Straftäterbehandlung. In: Bliesener T, Lösel F, Köhnken G (Hrsg) Lehrbuch der Rechtspsychologie. Huber, Bern, S 446-469

Hosser D, Greve W (2005) Jugendliche im Gefängnis - Strafhaft als Entwicklungsfolge und Entwicklungsbedingung. In: Schlottke P, Schneider S, Silbereisen RK, Lauth GW (Hrsg) Störungen im Kindes- und Jugendalter. Enzyklopädie der Psychologie, Bd. D/II/6. Hogrefe, Göttingen, S 655-680
Hosser D, Taefi A (2008) Die subkulturelle Einbindung von Aussiedlern im Jugendstrafvollzug. Monatsschr Kriminol Strafrechtsreform 91:131-143

Hosser D, Lauterbach O, Höynck T (2007) Und was kommt danach? Entlassungs-vorbereitung und Nachentlassungssituation junger Strafentlassener. In: Goerdler J, Walkenhorst P (Hrsg) Jugendstrafvollzug in Deutschland. Neue Gesetze, neue Strukturen, neue Praxis? Forum Verlag Godesberg, Mönchengladbach, S 396-412

Hosser D, Windzio M, Greve W (2005) Scham, Schuld und Delinquenz. Eine Rückfallstudie mit jugendlichen Strafgefangenen. Z Sozialpsychol 36:217-228

Hosser D, Windzio M, Greve W (2008) Guilt, shame and recidivism. Crim Justice Behav 35:138-152

Karstedt S, Greve W (1996) Die Vernunft des Verbrechens. Rational, irrational, banal: Die Rational-Choice-Theorien in der Kriminologie. In: Bussmann K-D, Kreissl R (Hrsg) Kritische Kriminologie in der Diskussion. Westdeutscher Verlag, Köln, S 171-210

Kazemian L, Walker A (2019) Effects of Incarceration. In: Farrington DP, Kazemian L, Piquero AR (Hrsg) The Oxford handbook of developmental and life-course criminology. Oxford University Press, Oxford, S 576-599

Klatt T, Bliesener T (2018) Evaluation des Jugendarrestes in Schleswig-Holstein. Forum Strafvollzug 67:320-326

Kuhl J (2001) Motivation und Persönlichkeit: Koalitionen psychischer Systeme. Hogrefe, Göttingen

Kuhl J, Hofmann F (2019) Diversität und Persönlichkeit: Begabungsentfaltung im Kontext der pädagogischen Beziehung. In: Reintjes C, Kunze I, Ossowski E (Hrsg) Begabungsförderung und Professionalisierung: Perspektiven, Konzepte, Herausforderungen. Julius Klinkhardt, Bad Heilbrunn, S 35-59

Laub J, Sampson R (2001) Understanding desistance from crime. Crime Justice 28:1-69

Laubenthal K (2019) Strafvollzug, 8. Aufl. Springer, Heidelberg, Berlin

Leipold B (2020) Intentional self-development and positive ageing. Routledge, London

Lerner RM (2002) Concepts and theories of human development. Erlbaum, Mahwah

Levinson DJ (1978) The seasons of a man's life. Random House, New York, Toronto

Levinson DJ (1986) A conception of adult development. Am Psychol $4: 3-13$

von Liszt F (1905) Strafrechtliche Aufsätze und Vorträge. DeGruyter, Berlin

Lösel F (1995) Meta-analytische Beiträge zur wiederbelebten Diskussion des Behandlungsgedankens. In: Steller M, Dahle K-P, Basqué M (Hrsg) Straftäterbehandlung. Centaurus, Herbolzheim

Lösel F (2018) Evidence comes by replication, but needs differentiation: the reproducibility issue in science and its relevance for criminology. J Exp Criminol 14:257-278

Lösel F (2020) Entwicklungspfade der Straftäterbehandlung: Skizzierte Wege und Evaluation der Zielerreichung. Forens Psychiatr Psychol Kriminol. https://doi.org/10.1007/s11757-020-00582-4

Lösel F, Bliesener T (1998) Risikofaktoren für die Aggressivität zwischen Jugendlichen. In: Kriminalistisches Institut des Bundeskriminalamts (Hrsg) Forum 1998. Bundeskriminalamt, Wiesbaden, S $137-159$

Lösel F, Bliesener T (2003) Aggression und Delinquenz unter Jugendlichen - Unter-suchungen von kognitiven und sozialen Bedingungen. Luchterhand, Neuwied

Lösel F, Link E, Schmucker M, Bender D, Breuer M, Carl L, Endres J, Lauchs L (2019) On the effectiveness of sexual offender treatment in prisons: a comparison of two different evaluation designs in routine practice. Sex Abuse. https://doi.org/10.1177/ 1079063219871576

Maelicke B, Suhling S (Hrsg) (2018) Das Gefängnis auf dem Prüfstand. Zustand und Zukunft des Strafvollzugs. Springer, Berlin 
Maruna S (2001) Making good. How ex-convicts reform and rebuild their lives. APA, Washington DC

McGee TR, Moffitt TE (2919) The developmental taxonomy. In: Farrington DP, Kazemian L, Piquero AR (Hrsg) The Oxford handbook of developmental and life-course criminology. Oxford University Press, Oxford, S 149-158

Mechler A (1981) Psychiatrie des Strafvollzugs. Fischer, Stuttgart

Merton RK (1938) Social structure and anomie. Am Sociol Rev 3:672-682

Meyer T, Greve W (2012) Die Entwicklungsbedingungen der Adaptivität: Theoretische Überlegungen und empirische Befunde zu einem Entwicklungsmodell akkommodativer Regulationskompetenz. Z Gesundheitspsychol 20:27-38

Moffitt TE (1993) Adolescence-limited and life-course-persistent antisocial behavior. Psychol Rev 100:674-701

Moffitt TE (2006) Life-course persistant versus adolescence-limited antisocial behavio. In: Cicchetti D, Cohen DJ (Hrsg) Developmental psychopathology. Wiley, New York, S 570-598

Morizot J, Kazemian L (Hrsg) (2015) The development of criminal and antisocial behavior. Springer, Berlin

Schmidt S, Bliesener T, van der Meer E (2016) Wie die kulturelle Sozialisation die Ausprägung und Vorhersagekraft von Risikofaktoren beeinflusst: Ein Vergleich erwachsener Strafgefangener ohne und mit türkischem bzw. arabischem Migrationshintergrund. Jahrbuch EFPPP 2016. Medizinisch Wissenschaftliche Verlagsgesellschaft, Berlin, S 94-104

Schmidt S, Bliesener T, van der Meer E (2019) Risk and protective factors of delinquency sensitive to migration and culture. J Psychol Crime Law. https://doi.org/10.1080/1068316X.2019.1597088

Schneider W, Lindenberger U (Hrsg) (2018) Entwicklungspsychologie, 8. Aufl. Beltz, Weinheim

Steller M (1983) Haftdauereinflüsse auf Selbstbeschreibungen von Delinquenten: Bezugsgruppeneffekte? Z Exp Angew Psychol 30:474-499

Stelly W, Thomas J (2005) Kriminalität im Lebenslauf. Eine Reanalyse der Tübinger Jungtäter-Vergleichsuntersuchung. TOBIAS-lib, Tübingen

Suhling S (2018) Wirkungsforschung und wirkungsorientierte Steuerung im Strafvollzug. In: Maelicke B, Suhling S (Hrsg) Das Gefängnis auf dem Prüfstand. Zustand und Zukunft des Strafvollzugs. Springer, Berlin, S 23-47

Suhling S, Greve W (2010) Kriminalpsychologie. Beltz, Weinheim
Suhling S, Neumann N (2015) Strafvollzugsforschung im Wandel? Positive Entwicklungen und Herausforderungen für Wissenschaft und Praxis. Kriminalpädag Prax 50:46-62

Taefi A, Hosser D (2019) Trajectories of delinquency among young adult prisoners. Eur J Criminol. https://doi.org/10.1177/ 1477370819877769

Thelen E, Smith LB (1998) Dynamic systems theories. In: Damon W, Lerner RM (Hrsg) Theorectical models of human development, 5. Aufl. Handbook of child psychology, Bd. 1. Wiley \& Sons, Hoboken, S 563-634

Thomsen T (2016) Flexible goal adjustment from late childhood to late adolescence: Developmental differences and relations to cognitive coping and emotion regulation. Int J Dev Sci 10:57-72

Thomsen T, Greve W (2013) Accommodative coping in early adolescence: an investigation of possible developmental components. J Adolesc 36(5):971-981

Ward T (2020) Why theory matters in correctional psychology: theoretical illiteracy and therapeutic ineffectiveness. Forens Psychiatr Psychol Kriminol. https://doi.org/10.1007/s11757-019-00578-9

Ward T, Gannon TA (2006) Rehabilitation, etiology, and self-regulation: the comprehensive good lives model of treatment for sexual offenders. Aggress Violent Behav 11:77-94

Weisburd D, Farrington DP, Gill C (Hrsg) (2016) What works in crime prevention and rehabilitation. Lessons from systematic reviews. Springer, New York

Wikström P-OH (2014) Why crime happens: a situational action theory. In: Manzo G (Hrsg) Analytical sociology: actions and networks. Wiley \& Sons, Hoboken, S 74-94

Wischka B, van den Boogaart H (2018) Sozialtherapie im Justizvollzug. In: Maelicke B, Suhling S (Hrsg) Das Gefängnis auf dem Prüfstand. Zustand und Zukunft des Strafvollzugs. Springer, Berlin, S 129-157

Wrosch C, Heckhausen J (2005) Being on-time or being off-time: developmental deadlines for regulating one's own development. In: Perret-Clermont A-N (Hrsg) Thinking time: a multidisciplinary perspective on time. Hogrefe, Göttingen, S 110-123

Wrosch C, Scheier MF, Miller GE, Schulz R, Carver CS (2003) Adaptive self-regulation of unattainable goals: goal disengagement, goal reengagement, and subjective well-being. Pers Soc Psychol Bull 29:1494-1508 